Káñina, Rev. Artes y Letras, Univ. Costa Rica XXXVIII (2): 91-125, 2014 / ISSN: 2215-2636

\title{
OPUSCULE SUR LES NOUVELLES HÉROÏQUES ET AMOUREUSES DE BOISROBERT
}

\author{
Opusculum about Boisrobert's Nouvelles hérö̈ques et amoureuses
}

\section{Francisco Guevara Quiel*}

\begin{abstract}
RÉSUMÉ
En 1657, l'abbé Boisrobert, membre fondateur de l'Académie française, fait paraître un recueil de nouvelles peu connu. La critique ne s'est pas penchée jusqu'à présent sur l'intérêt majeur qu'elles représentent du point de vue du processus d'adaptation opéré à travers des sources espagnoles et françaises à la fois, et sur le fait qu'elles se font écho d'une idéologie culturelle en crise à la charnière entre la période dite « baroque » et la période dite « classique ». Cet article pose alors le triple enjeu de mettre en lumière l'existence de cette œuvre oubliée, montrer sa portée en termes d'adaptation, et réfléchir sur les enjeux éthiques et esthétiques dans le passage d'une littérature nationale à une autre, dans le cadre d'un mouvement de vaste ampleur au milieu de XVIIe siècle français.
\end{abstract}

Mots-clé: Boisrobert, nouvelles, adaptation, traduction, Siècle d'or.

\section{RESUMEN}

En 1657, el abad Boisrobert, miembro fundador de la Academia francesa, publica un compendio de novelas cortas poco conocido. Hasta ahora la crítica no ha percibido el interés mayor que estas representan en cuanto al complejo proceso de adaptación realizado a través de fuentes españolas y francesas a la vez, y al hecho reflejar una ideología cultural en crisis en la transición del barroco al clasicismo. Este artículo plantea así el triple desafío de poner en valor la existencia de esta obra olvidada, mostrar el alcance del trabajo adaptativo en términos de literatura comparada, y reflexionar sobre los desafíos éticos y estéticos a los que da lugar la adaptación de una literatura nacional a otra, dentro de un movimiento de mayor amplitud en la primera mitad del siglo XVII francés.

Palabras clave: Boisrobert, novelas, adaptación, traducción, Siglo de oro.

\begin{abstract}
In 1657, abbot Boirobert, founder member of the Académie française, publishes a little known collection of short stories. The criticism did not deal with the major interest that they represent contents of a complex process of adaptation operated from French and Spanish sources at the same time, and that it sends back to a cultural ideology in the hinge between the baroque and the classicism. This article thus puts the triple challenge to assert the existence of a forgotten work, show the reach of the adaptive work in terms of compared literature, and think about the ethical and esthetic stakes which raises the question of the adaptation of a national literature to an other one within the framework of a large-scale movement taking place in the first half of the French XVIIth century.
\end{abstract}

Key Words: Boisrobert, novels, adaptation, translation, Golden Age.

Universidad de Costa Rica, Escuela de Lenguas Modernas, Costa Rica.

Correo electrónico: fguevaraquiel@yahoo.com

Recepción: 23/1/2014 Aceptación: 27/3/2014. 
Nous avons déjà abordé quelques aspects généraux sur Les Nouvelles hérö̈ques et amoureuses ${ }^{1}$ de l'abbé Boisrobert dans un article précédent ${ }^{2}$, où l'on s'est interrogé notamment sur les enjeux esthétiques de cette œuvre en termes d'adaptation à partir d'une série de sources espagnoles (et/ou françaises) diverses et variées tant en mode narratif que dramatique. Pourtant la plupart des questions qui y ont été posées est restée en attente de réponse et ces questions tenaient précisément à ces aspects-là, contenu du fait qu'il manquait des vérifications à faire. S'il est vrai que les sources de ces Nouvelles ont été en partie repérées par la critique savante, avec assez de certitude pour deux d'entre elles, il est vrai aussi que pour les deux autres, la recherche n'offre que de pistes assez floues ou approximatives sans que pour autant elles soient irrecevables. Au contraire. Quand on constate qu'elles semblent moins simples à cerner qu'il ne paraît et qu'elles appellent à une fixation et à une attention tout particulières sur quelques textes littéraires et historiques, on perçoit bien que Boisrobert a pu procéder par un complexe processus de contamination qui rend la tâche assez délicate et que nous tenterons de démêler ici.

Deux aspects nous retiennent donc à ce stade;: d'abord, la réalisation d'un état présent de la question - le plus précis possible - des sources susceptibles d'intéresser ces nouvelles françaises, et ensuite l'établissement de certaines considérations par rapport à ce qu'elles peuvent représenter du point de vue esthétique.

\section{Les sources}

Chardon se plaisait à dire que D’Ouville, frère de l'abbé, avait laissé un portefeuille avec des œuvres à publier et que "Boisrobert vint au Mans, je ne dis pas pour le détrousser, mais pour profiter de ses manuscrits. D'ailleurs ne les a-t-on pas longtemps considérés comme collaborant ensemble, D'Ouville fournissant les plans des comédies et laissant à Boisrobert le soin d'en composer les vers ${ }^{3}$ ?». C'est luimême qui rapporte que le facétieux Tallemant des Réaux disait que Boisrobert: «s'est amusé à cajoler le libraire pour tirer cent livres de quatre nouvelles espagnoles qu'il a mises en mauvais français ${ }^{4} \gg$, pour conclure que ce sont des $«$ traductions $^{5}$ » qui provenaient des papiers de feu son frère.

Mais outre que Chardon n'indique pas la source de ce commentaire, on est là dans la spéculation pure et simple. On reconnaît bien dans ce genre de propos ce qui constitue l'attitude généralement suivie par presque toute la critique littéraire touchant le cas de Boisrobert depuis le XVII ${ }^{\mathrm{e}}$ siècle, sans doute par une facilité d'esprit à tous égards commode. Chardon non seulement omet de signaler l'origine de ces rumeurs mais se fait écho impassible des propos désinvoltes de Tallemant, lesquels on a souvent trop pris pour de l'argent comptant. On regrettera donc qu'ils prennent le pas sur la production littéraire de l'auteur et qu'ils n'apportent rien à la connaissance et à la compréhension de celleci, n'ayant pas contribué à avancer d'un pouce dans l'étude objective, cohérente et systématique de cette œuvre. Boisrobert est de ces auteurs qui ont eu le malheur de soulever d'acerbes critiques (souvent gratuites) de la part de ses contemporains $^{6}$, dont énormément d'attaques personnelles qui ont mis un voile sur son travail littéraire. À croire que sa personnalité exerçait une fascination indéniable et plus forte, et sur laquelle on s'obstine à s'arrêter éclaboussant (négativement) l'œuvre. On aurait voulu surtout savoir à quoi tient le discrédit de celle-ci, si tant est qu'elle le mérite. Et indistinctement des travers ou des vertus de Boisrobert, il conviendrait plus de se pencher sur ce qu'il a écrit, sur la provenance de ce matériau au regard de ce qu'il en dit dans les paratextes, sur ce qu'il en a fait et pourquoi, sans quoi il serait difficile de comprendre en quoi cette œuvre peut être cohérente et relever d'une vraie esthétique de l'adaptation. C'est pourquoi nous essaierons par la suite de nous arrêter sur chaque nouvelle en particulier par rapport aux sources et ensuite sur leur caractère esthétique.

On constate d'abord que les auvres contenues dans ce recueil - lequel constitue l'une des dernières manifestations littéraires de l'auteur - sont au nombre de quatre, et par 
ordre de succession : L'Heureux Désespoir (p. 1-179), L'Inceste supposé (p. 183-308), Plus d'effets que de paroles (p. 309-438), et La Vie n'est qu'un songe (p. 439-550). La numérotation continue des pages montre bien qu'il ne s'agit pas d'un recueil factice mais d'un projet conçu et concrétisé comme un ensemble. Elles n'ont d'ailleurs pas fait l'objet d'une publication individuelle préalable, comme ce fut le cas pour les nouvelles de Scarron, de D'Ouville et d'autres écrivains. En termes de longueur, les textes restent relativement proportionnés, avec une moyenne de 161 pages, mais si $L a$ Vie n'est qu'un songe atteint les 211, L'Inceste supposé en compte à peu près la moitié. C'est dire qu'on reste dans des longueurs raisonnables pour ce genre littéraire par rapport aux romans fleuves qui atteignent parfois plusieurs volumes, quoique l'extension ne constitue pas forcément un critère de classification pour les uns et les autres. René Godenne remarque d'ailleurs qu'au temps où Boisrobert fait paraître son recueil, cinq ou six histoires suffisent pour en former un et que la longueur moyenne varie entre les deux à trois cents pages dans un format in $-8^{\circ}$, mais il y a des cas qui dépassent facilement les sept cents, ce qui fait que certaines nouvelles sont publiées séparément. On retrouve ici en quelque sorte une pratique qui rejoint l'esthétique romanesque des auteurs du premier XVII ${ }^{\mathrm{e}}$ siècle ${ }^{7}$.

\section{A. Repérages critiques}

Des études critiques modernes comme celles d'Antoine Adam ${ }^{8}$, Henry C. Lancaster ${ }^{9}$, Alexandre Cioranescu ${ }^{10}$, Roger Guichemerre ${ }^{11}$ et José Manuel Losada Goya ${ }^{12}$, nous ont apporté de précieuses informations pour l'identification des sources de la plupart des œuvres de Boisrobert lorsque celui-ci ne les indique pas ${ }^{13}$. Il faut dire que la somme publiée par Lancaster constitue le support principal à partir duquel nous avons pu repérer la plupart d'entre elles. L'auteur en fait une analyse succincte et les place dans leur contexte de production. Il donne aussi un résumé et des commentaires d'ordre dramaturgique tout en établissant des liens avec d'autres pièces de théâtre ou avec des genres littéraires différents, comme la nouvelle ou le roman, avec lesquels les œuvres de Boisrobert ont en effet des rapports directs ou indirects. Il propose enfin des hypothèses sur la source possible d'une pièce lorsqu'elle n'est pas avérée, et en ce sens, il ouvre la perspective de recherche en envisageant des pistes alternatives.

Concernant le récent travail bibliographique de Losada Goya, dont le point de départ est la somme de Lancaster, il se distingue de celle-ci en ce qu'il cherche à donner un aperçu sur les sources des œuvres françaises en prose, ce que le critique américain ne pouvait pas envisager ayant consacré son étude uniquement au théâtre du XVII ${ }^{\mathrm{e}}$ siècle. Mais s'agissant des sources des nouvelles de Boisrobert, Losada Goya se limite à faire le point sur ce qu'ont déjà dit essentiellement Cioranescu et Guichemerre, car sa Bibliographie ne consiste pas vraiment en un travail d'analyse mais de synthèse.

Ainsi, nous avons pu recenser un total de quinze sources espagnoles, partagées entre comedias et novelas, pour l'ensemble des œuvres de l'abbé. La critique montre que sept pièces de théâtre et une nouvelle ont donné lieu à huit comédies $^{14}$, tandis que pour les trois tragicomédies de cette période, deux d'entre elles sont directement issues de pièces de théâtre espagnoles ${ }^{15}$ et la dernière résulte de l'adaptation d'une nouvelle du même Boisrobert ${ }^{16}$, nouvelle qui à son tour, ainsi que l'abbé l'indique en termes peu précis dans la dédicace de l'œuvre, est un sujet «tiré tout nu de l'Espagnol». L'auteur a également écrit un recueil de nouvelles, Les Nouvelles héröques et amoureuses, qu'il présente comme étant tirées d'une source hispanique. Nous y reviendrons.

\section{B. Boisrobert adaptateur de la littérature espagnole en France}

À ce stade une mise en contexte des œuvres de l'abbé devient nécessaire. On va pouvoir distinguer deux étapes bien délimitées dans la carrière littéraire de notre auteur, où certains événements ont une influence directe sur sa production ${ }^{17}$. La première phase est celle où il vit sous la protection de Richelieu, qui 
institutionnalise la pratique du clientélisme et dans le cadre de laquelle Boisrobert jouit de privilèges exceptionnels; la seconde, qu'Alain Viala appelle «stratégie du succès», à défaut de mécène, repose sur la capacité de l'auteur à faire valoir sa propre production littéraire auprès du public. Il s'agit pour lui d'assumer pleinement sa condition de professionnel de lettres, ou plus particulièrement d'auteur dramatique et narratif malgré son état ecclésiastique, par principe incompatible avec l'activité théâtrale.

À cette période, l'influence de la Comedia espagnole se fait vivement sentir sur la scène littéraire française et la stratégie du succès cherche à s'appuyer sur celle-ci pour remporter l'approbation d'un public le plus large possible, ce qui contribue à établir la réputation des auteurs dans le domaine dramatique et narratif. Ainsi, tout en cherchant à se procurer la protection d'une personnalité distinguée de la vie politique et sociale, Boisrobert fait «l'épreuve» de ses créations dans les cercles mondains (cf. la dédicace de la Cassandre, à titre d'exemple) avant de les donner au public sur le théâtre ou sur le papier. La pratique était d'ailleurs largement répandue et souvent efficace pour s'attirer la bienveillance de l'auditoire sensibilisé par avance aux ouvrages. Ajoutons qu'après la disparition de Richelieu et jusqu'au moment où l'auteur rentre dans sa «période espagnole», son statut est particulièrement flou en société. Car s'il est vrai qu'il reste abbé de Châtillon, on le retrouve bien davantage débitant vers et prose dans les ruelles que sur la chaire, et s'il fait partie de la récente Académie - où il n'est pas certain qu'il ait beaucoup siégé -, rien ne permet de constater qu'il ait beaucoup participé à ses débats. Enfin, Boisrobert n'est pas non plus attaché à la clientèle d'un mécène. Sa stratégie du succès s'appuie donc fondamentalement sur le public galant de l'époque, essentiellement parisien (ne jouissant pas trop des grâces de la cour), auquel il adresse sa production. Cette œuvre obéit ainsi, en partie, à une nécessité immédiate de reconnaissance sociale et s'inscrit dans un mouvement en vogue dont il voulut tirer le meilleur parti possible.

\section{De l'adaptation des Nouvelles héröques}

C'est ce qui nous permet par la suite de nous occuper de l'étude particulière de chacun des récits qui intègrent ce recueil de Boisrobert et à partir desquels nous essaierons de montrer les adaptations faites par l'auteur, ce qu'il prend et ce qu'il laisse de côté, toute en s'interrogeant par rapport à la cohérence de l'œuvre, et après avoir indiqué les sources que nos recherches et la consultation des références critiques citées nous ont permis de repérer.

\section{L'Heureux Désespoir}

Un cas particulier du travail sur les sources fait par Boisrobert est celui de sa première nouvelle, L'Heureux Désespoir. La critique a consacré quelques études à l'œuvre qui est à l'origine du récit de notre abbé, la tragi-comédie L'Heureuse Constance de Rotrou, publiée en 1636. Il s'agit donc d'une pièce française à laquelle il a eu recours, contrairement à ce que l'auteur nous avait annoncé dans la dédicace à Nicolas Foucquet, et non (directement) à une comedia espagnole. Mais il est à noter que l'œuvre en question de Rotrou résulte de la combinaison par contamination de deux comedias de Lope de Vega à la fois, à savoir El poder vencido y amor premiado $^{18}$ (1618) et Mirad a quién alabáis ${ }^{19}$ (1621). Georges Hainsworth ${ }^{20}$, Katherine King ${ }^{21}$ et Sven Birkemeier ${ }^{22}$ figurent parmi ceux qui ont bien établi les rapports entre ces deux pièces et celle du dramaturge originaire de Dreux. Boisrobert pour sa part, utilise la tragi-comédie de Rotrou, mais en bon «tailleur», il a réussi à la déguiser parfaitement d'un somptueux cadre mauresque de la fin du Moyen Âge espagnol, transpososant l'intrigue dans la cour du dernier roi nasride de Grenade, Boabdil.

Ainsi, tandis que G. Hainsworth déclarait dans un premier ouvrage ${ }^{23}$ que la source de la nouvelle de Boisrobert était inconnue, dans un article paru quatorze ans plus tard ${ }^{24}$, il déclare que L'Heureuse Constance est simplement remise au goût mauresque, alors que Rotrou avait transposé l'intrigue en Hongrie et en Dalmatie. 
En effet, notre abbé a recours à la pseudohistoire de Ginés Pérez de Hita, Guerras civiles de Granada, où l'on retrouve le même décor que dans des récits de tradition mauresque espagnols, comme El moro Abindarráez y la bella Jarifa (novela anonyme rendue célèbre dès sa publication dans La Diana de Montemayor), et Osmín y Daraja (novela intercalée dans la première partie du Guzmán de Alfarache de Mateo Alemán), où il est question de la rivalité d'un roi avec son frère et des thèmes hispanomauresques. En France, on peut citer une pièce de Quinault, La Généreuse Ingratitude, tragicomédie pastorale parue en 1654, où le thème mauresque est travaillé, et peut-être cette pièce a-t-elle influencée Boisrobert, ne serait-ce que pour le nom du roi Almanzor, que l'on ne trouve pas dans le récit de Pérez de Hita et qu'il fallait que Boisrobert «invente» puisque chez Rotrou, on le désigne simplement sous le nom de «roi» (à la scène sept de l'acte IV, il signe une lettre adressée à son frère Alcandre sous le nom de Fernand).

Dans L'Heureuse Constance, le roi de Hongrie, engagé en mariage avec Arthémise, reine de Dalmatie, se déguise en « villageois » pour la connaître avant leur rencontre officielle et savoir si elle lui plaira. Mais tout juste avant que cela n'arrive, il tombe, dans cet accoutrement, éperdument amoureux d'une «paysanne» nommée Rosélie qu'il aperçoit passant près de lui comme un éclair. Alors, il charge Timandre, son fidèle courtisan, de savoir qui elle est et de la lui ramener. Timandre, qui ne savait pas que la dame déguisée était sa propre sœur, doit, embarrassé, lui avouer cela après l'avoir reconnue et qu'il avait été contraint de la cacher dans ce village apprenant ses amours secrètes avec Alcandre, frère du roi. Puis, prévoyant le double danger que court Rosélie (l'amour du roi et d'Alcandre), Timandre propose à sa sœur d'épouser Pâris, ambassadeur près de la reine Arthémise, qu'il doit emmener bientôt en Hongrie. Mais aussitôt arrivé, Pâris reçoit l'ordre royal de reconduire la reine chez elle dans ses États dalmates puisque le roi ne souhaite plus l'épouser. La souveraine, furieuse de cet affront, est obligée de rentrer dans son pays, tandis que subjugué par Rosélie, Fernand vient même à lui proposer sa couronne au désespoir d'Alcandre qui en est témoin, mais elle lui donne une réponse évasive et promet à son amant de rester fidèle. Entre temps, Arthémise arrive en Dalmatie désireuse de se venger au plus vite de l'humiliation essuyée et s'apprête à lever une puissante armée contre le roi. Elle offre aussi sa main à Pâris qui trouve du charme à ses yeux, mais cet ambassadeur, épris aussi de Rosélie, trouve les mots justes pour refuser cette honorable offre royale. Fernand, qui apprend les amours d'Alcandre et Rosélie, ainsi que les projets belliqueux de la reine, ordonne à son frère de se rendre à la cour d'Arthémise pour l'épouser et apaiser ainsi sa colère. Alcandre exécute malgré lui les ordres irrévocables de son frère, mais il a recours à la ruse à l'aide de son valet bouffon Ogier, qui ourdit le stratagème d'emprunter des habits de prince pour se faire passer par lui et se présenter devant la souveraine. L'idée est de la rebuter en regardant un prétendant aussi extravagant que ridicule, et la ruse produit l'effet souhaité. Pourtant la reine aurait souhaité que ce serviteur du prince (Alcandre lui-même) eût été celui qu'on lui eût envoyé. Pour s'assurer de la séparation des amants, le roi invente un autre subterfuge qui consiste à envoyer de fausses lettres à Alcandre et à Rosélie, par lesquelles ils renoncent à l'amour qu'ils s'étaient promis. La ruse est pourtant déjouée, car les amants se revoient et s'expliquent. Le roi les surprend pendant cette entrevue, et dans un élan de colère, il fait enfermer son frère en prison. Arthémise, curieuse de connaître la beauté tant célébrée de Rosélie, et dont on disait des merveilles, se rend en Hongrie secrètement déguisée en " pèlerine ", et accède à sa chambre même pour l'entretenir. Pâris survient qui reconnaît la reine déguisée, et ensuite le roi, qui en regardant le visage de la reine, en présence de Rosélie, en tombe éperdument amoureux. Apprenant qu'il s'agit d'Arthémise, il la supplie de lui pardonner et d'accepter sa main. Ainsi, «l'heureuse constance» de Rosélie et d'Alcandre est récompensée par l'autorisation du roi à se marier; Pâris aussi reçoit la main de Florinée, 
«parente» de la reine et à la fin, trois mariages peuvent avoir lieu.

Dans la nouvelle de Boisrobert, l'intrigue reste la même malgré quelques changements. Le roi Almanzor est de même sur le point d'épouser une reine qu'il ne connaît pas, Chériffe, princesse de Fez, mais lorsqu'il assiste à un tournoi magnifique, il aperçoit une jeune beauté en habits de paysanne dont il tombe éperdument amoureux. Alors, il charge Almazan, son fidèle courtisan, de savoir qui est cette belle, et Almazan avoue qu'il s'agit de sa propre sœur qu'il cachait dans le village où se réalise le tournoi, ayant appris les amours secrètes qu'elle entretient avec Abindare, frère du roi. Cette jeune fille n'est autre que Darache, qui n'avait trouvé que ce moyen pour aller voir son amoureux déployer tous ses talents de cavalier dans la course. Pour éviter le double danger qui menace Darache (l'amour du roi et d'Abindare), Almazan propose à sa sœur d'épouser Alabez, ambassadeur près de la reine Chériffe, qu'il doit bientôt emmener à Grenade. Comme le roi apprend que son frère en est amoureux et en est aimé, il lui propose d'épouser la princesse de Fez, avec laquelle il était engagé en mariage, puisqu'il est désormais subjugué par Darache, à qui il propose sa couronne en présence d'Abindare, sans qu'il obtienne une réponse favorable. Entre temps, Chériffe arrive sur les côtes d'Almerie, où elle apprend que le roi se délie de ses engagements. Furieuse pour cette humiliation, elle menace de mettre Grenade à feu et à sang si le roi ne l'épouse pas. En bon diplomate, Alabez parvient à lui proposer la main du prince Abindare en compensation. Celui-ci est contraint d'accepter sous la pression de la noblesse pour ramener le calme au royaume, vu que la reine fait des ravages sur les côtes du pays ; mais le prince a recours à la ruse, car il fait que son valet Abenamar emprunte ses habits et son nom pour se présenter devant la souveraine, qui voyant ce prétendant si ridiculement accoutré et extravagant refuse absolument de l'épouser, tel qu'ils l'avaient prévu. Mais elle apprend aussitôt qu'on vient de lui jouer un mauvais tour, ce qui redouble sa rage et la détermine davantage à continuer la destruction du royaume. Cependant, comme elle tombe amoureuse d'Abindare lorsqu'elle le voit, elle menace de détruire Grenade si on ne le lui donne pas pour époux. Outré par l'idée de perdre Darache, il essaie de se suicider. On amène Darache auprès de la reine, qui touchée par sa beauté éblouissante et par le grand amour que ces jeunes gens s'avouent, renonce à ses prétentions et donne la liberté au prince d'épouser cette belle. Le roi, quant à lui, ne survivra pas longtemps. Ainsi, «on doit admirer la constante amour du Prince Abindare $\&$ son heureux desespoir», comme l'exprime Boisrobert à la fin de sa nouvelle.

On remarque ainsi que Boisrobert suit de très près la pièce de Rotrou en faisant quelques ajustements à l'intrigue, les plus importants concernant les apparitions de la reine et le fait qu'elle épouse le roi à la fin de la pièce, ce qui n'arrive pas chez Boisrobert, qui finit son récit sur un registre plutôt grave et tragique. De même, si l'on ne sait pas combien de temps nécessite la pièce de Rotrou pour être dénouée, on voit que Boisrobert a le souci de concentrer le récit le plus possible dans le temps et dans l'espace; il ne nous déplace pas par exemple au Maroc, ce que fait Rotrou avec Pâris et Arthémise en les promenant en Dalmatie et en Hongrie. Chez Boisrobert, on est tantôt au port d'Almerie, tantôt dans la cour Nasride, mais on reste toujours dans des proximités géographiques à l'intérieur du royaume nasride. L'on peut dire enfin qu'il a introduit un épisode de la nouvelle Ozmín et Daraja ${ }^{25}$ où le prince se déguise en jardinier pour pouvoir s'entretenir avec son amoureuse, à cause de la difficulté que fait Almazan pour qu'elle rencontre son amant. Chez l'abbé, on voit aussi l'amant désespéré chercher toute sorte de moyens pour revoir la belle Darache, qui est fortement surveillée par la sévère Fatime, sa gouvernante et espionne au service d'Almazan. Il soudoie le jardinier Bilbar, qui travaille au service de celui-ci, pour s'introduire chez la belle prisonnière. Concernant l'univers arabesque, on constate que l'auteur réussit parfaitement à nous situer dans un cadre géographique mauresque par la série de données, très précises, que l'on trouve tout au long du récit et qui sont véridiques, comme par exemple, les 
rivières de Genil ou de Darro qui traversent la cité-État, ou certaines villes de l'Andalousie comme Huéscar et Caniles, ou encore les noms des palais qui conforment la ville-forteresse de Grenada, à savoir l'Albaicín, les Torres Bermejas et l'Alhambra. Boisrobert cherche à nous imprégner aussi de l'histoire de Pérez de Hita quant aux clans qui règnent à Grenade, celui des Alabez, des Zegríes, des Nasrides et des Abencerrajes, ainsi que sur leurs rapports avec les grandes familles parentes de Tlemcen et de Fez. En réalité, il s'agit d'un important travail de recherche, comme il a fait également par rapport aux autres nouvelles. Le lecteur est ainsi vraiment dépaysé dans un cadre exotique précis, mais il plane sur des imprécisions historiques et temporelles qui nous renvoient au caractère très romanesque de l'intrigue. Il est clair qu'il ne s'agit pas pour Boisrobert de jouer le rôle d'historien. L'on comprend ainsi que la critique ait eu beaucoup de mal à trouver la source de cette nouvelle, où notre «tailleur» parvient à exécuter un complexe travail d'adaptateur.

Pour cette nouvelle, on pourrait donc parler d'un Boisrobert plutôt lecteur de Rotrou que d'auteurs espagnols. Il est clair qu'il ne devait pas ignorer que la pièce du Français remonte à Lope de Vega, dont l'abbé ne tarissait pas d'éloges et qu'il considérait comme un grand inventeur mais qui au milieu du $\mathrm{XVII}^{\mathrm{e}}$ siècle n'était plus un créateur suivi en France, à cause de l'extrême irrégularité qu'on lui attribuait. Par ailleurs, Rotrou avait déjà opéré un travail de synthèse considérable et commode dans L'Heureuse Constance qui satisfaisait parfaitement les exigences classiques de concentration et dont Boisrobert a manifestement tiré partie. Facilitant donc la tâche d'adaptation, celui-ci est allé plus loin en termes d'inventio et de dispositio pour faire en sorte que son récit soit le plus possible conforme aux normes. En revanche, quel intérêt peut présenter ce sujet, a priori peu édifiant et plutôt peuplé de péripéties et d'aventures héroïques tant appréciées dans la première moitié du siècle mais plus tellement à la mode en 1658 , si ce n'est que du côté de l'image qu'il offre de la femme ? Car en effet, bien que la peinture psychologique soit quasiment inexistante, nous avons deux héroïques qui se détachent de l'ensemble soit par la beauté sublimée de l'une (Darache) soit par la magnanimité et la générosité de l'autre qui appellent à l'admiration et à la révérence (la reine). Deux «modèles» de femmes donc qui devaient être ou à l'image de la femme à laquelle Boisrobert idéalement s'adresse, ou alors au goût encore présent et fort pour ce genre de caractères auquel est sensible une partie de son lectorat, notamment féminin, celui des ruelles galantes. On ne sait que trop que l'abbé est connu comme le "grand prêtre des ruelles", qu'il y trône en " dupeur d'oreilles » et qu'il anime les cercles régentés par quelques dames distinguées. On a du mal encore à mesurer l'impact des ouvrages de ce genre du "plaisant abbé» mais il n'est pas anodin et sans intérêt de constater qu'il continue à nourrir sans aucun doute un goût encore présent en société. En tous les cas, s'agissant de la source espagnole, elle est bel et bien existante. Boisrobert n'a pas dit toute la vérité dans sa dédicace, du moins il ne l'a dite qu'à moitié, car il n'a pas totalement menti. Mais peut-on lui reprocher ce manque de précision et le fait de nous laisser croire éventuellement que sa nouvelle est directement adaptée d'une œuvre hispanique?

\section{L'Inceste supposé}

Autrement complexe est le cas de sources multiples, directes et indirectes, pour cette nouvelle de Boisrobert. Il s'agit de la seconde du recueil des Nouvelles héroïques et amoureuses: L'Inceste supposé, œuvre dont l'origine n'est pas sans poser difficulté en ce sens qu'il existe déjà une pièce française, publiée dès 1640 par La Caze, qui porte le même titre et dont l'intrigue est proche de celle-ci. Or il se trouve que le sujet de cette nouvelle est aussi le même que celui de la dernière tragi-comédie de Boisrobert, Théodore, reine de Hongrie, pièce que l'on sait avoir été la cible de la critique la plus acerbe que l'on ait vue au XVII siècle à l'encontre de la personne de Boisrobert et de cette œuvre en particulier. Il s'agit des fameuses Remarques sur la Théodore de l'auteur de Cassandre, écrites 
par Antoine Baudeau de Somaize et parues le 6 août 1657. C'est dans ces Remarques que Somaize affirme, à l'appui de preuves concrètes, que Boisrobert aurait pillé la pièce de La Caze, publiée en 1640. Le fait est que, comme nous l'avons déjà signalé, si les Nouvelles héröques virent le jour le 12 mai 1657, la Théodore ne fut jouée que le 15 juin 1657, tandis que le texte de la pièce ne fut publié que le 15 novembre de la même année. Il y a donc six mois d'intervalle entre les nouvelles et le poème dramatique, ce qui nous induit à penser que les nouvelles furent écrites bien avant la pièce. Et c'est là un aspect que la critique n'a jamais examiné de près jusqu'à présent ${ }^{26}$. C'est ce qui pose des difficultés pour déterminer exactement l'origine des sources. En toute logique, il faudrait aborder cette question par rapport à un ordre chronologique, d'autant que l'on constate que le sujet de la nouvelle et celui de la pièce sont strictement les mêmes.

D'abord, on peut voir dans la dédicace à Foucquet du recueil que Boisrobert se prononce clairement à propos de cet aspect en ces termes:

\begin{abstract}
Si les plus beaux Esprits du siecle à qui j'ay communiqué ces Nouvelles, avant que d'oser les publier sous l'authorité de vostre nom, ne m'ont point flatté, les sujets que j'ay tirez tous nuds \& tous simples de l'Espagnol, \& que j'ay rectifiez selon nos manieres, en sont tout particuliers \& tout beaux, les incidens en sont tout merveilleux $\&$ tout surprenans, le stile en est pur $\&$ net, $\&$ enfin ce petit ouvrage peut passer parmy les plus delicats de la Cour pour galand $\&$ pour agreable. (1. 1-5).
\end{abstract}

On va cependant constater que ces lignes de Boisrobert ne sont qu'à moitié vraies, surtout lorsqu'on vient de voir que la toute première nouvelle du recueil n'est pas tirée directement d'une œuvre espagnole mais d'une tragicomédie de Rotrou, L'Heureuse Constance, que notre auteur a habilement travesti d'une histoire hispano-mauresque provenant des Guerras civiles de Granada, de Ginés Pérez de Hita. Et s'il est vrai que la troisième et la dernière sont, elles, directement tirées de comedias, la seconde, L'Inceste supposé, nous laisse penser que l'auteur a eu effectivement recours à une œuvre espagnole, même s'il faut tenir en considération le fait qu'il ait pu aussi utiliser la tragi-comédie de La Caze, ne serait-ce qu'à titre de référence. Car ce sont d'abord les dates de diverse nature qui nous renseignent sur la chronologie dans la parution de ces œuvres.

En effet, la tragi-comédie de La Caze fut publiée dix-sept ans plus tôt, en 1640, tandis que La perseguida triunfante, appartenant au recueil Los desengaños amorosos de María de Zayas Sotomayor ${ }^{27}$, qui nous semble être la source directe de cette nouvelle de Boisrobert, parurent pour la première fois en 1647. Manifestement La Caze n'a donc pas pu se servir de cette nouvelle espagnole. En revanche, en comparant celle-ci avec L'Inceste supposé de Boisrobert, nous avons trouvé que notre auteur aurait pu s'appuyer suffisamment sur La perseguida sans avoir nécessairement besoin d'autre source, car le matériau de l'œuvre espagnole lui offre tous les éléments requis pour élaborer sa propre intrigue en suite d'un considérable travail de synthèse et de concentration. Il est évident que l'histoire racontée par María de Zayas est parfaitement conforme au genre (dans la tradition du conte boccacien) et à la finalité du mode narratif, et qu'elle n'a cure d'organiser son intrigue sur un quelconque modèle ou programme dramaturgique préalable (du moins rien ne le prouve). Elle commence donc $a b$ ovo et non in medias res, comme la tragicomédie de Boisrobert. Elle est aussi largement plus longue, plus fantaisiste, plus fantastique et baigne dans le merveilleux; elle est pleine de rebondissements, d'aventures et d'épisodes des plus cruels aux plus attendrissants, le sang coule à flots, les tentatives de viol sont à l'ordre du jour, les meurtres et les outrages les plus barbares se succèdent les uns après les autres; on trouve aussi des sortilèges à foison, des déités et des démons qui s'évanouissent et réapparaissent au gré des circonstances et à point nommé pour secourir la bonne ou le méchant de l'histoire, bref des cabales, des jalousies, des calomnies, des injustices en tous genres et tout ce qui peut agrémenter un récit où la femme écrivain s'efforce d'exalter la figure féminine et de dénoncer la barbarie, l'injustice et la violence faites par la gent masculine contre celle-ci. Outre le plaisir esthétique de la prose de 
María de Zayas, son œuvre se présente comme porteuse d'un message clairement adressé à ses congénères, et comme un signal d'alerte à la fragile condition de la femme exposée à tant de risques et de méfaits dans un monde gouverné exclusivement par les hommes. Ce sujet ne pouvait que trop plaire et convenir à Boisrobert, comme on le verra.

En voici l'histoire: Ladislao, roi de Hongrie, épouse par procuration la princesse Beatriz que son frère cadet Federico va chercher personnellement en Angleterre. Celui-ci en tombe passionnément amoureux, la princesse s'en aperçoit mais fait semblant de ne rien comprendre et se fait toujours accompagner de ses suivantes pour éviter toute éventuelle inconduite de la part du jeune homme. En Hongrie, les noces sont officiellement célébrées, mais Federico persiste en son amour « incestueux " même un an après ce mariage. C'est alors que le roi doit quitter le palais pour se battre contre les ennemis dans les frontières du royaume, donnant ainsi l'occasion à son frère de séduire la chaste épouse. Il s'évertue ainsi à déclarer sa flamme amoureuse par lettre, que la reine lit avec un extrême déplaisir et déchire en colère. Pourtant elle n'en dit rien, ce que le prince interprète comme un consentement tacite à ses avances, osant même lui chanter des sérénades et lui baiser sensuellement la main à un moment donné. Pour arrêter ses égarements, elle décide de construire une cage en fer pour l'enfermer, ce qui fait que l'amour qu'il lui porte se transforme soudainement en une haine mortelle. Dès lors, il ne songe qu'à se venger, et l'occasion se présente lorsque son frère annonce son retour, allant devant lui et devançant Beatriz pour la calomnier tout en renversant la vérité des faits: alors il dit au roi qu'elle l'a effrontément séduit, puis enfermé pour refuser d'assouvir ses désirs charnels. Ladislao croit aveuglement la version donnée par son frère, sans examiner un instant si sa femme était vraiment coupable de cela. Il lui fait donc un procès sommaire, la punit en la giflant violemment et la condamne à errer dans une forêt pour être dévorée des bêtes sauvages, une fois que ses cruels soldats lui aient arraché les yeux. Mais à ce comble d'infamie, la reine est aussitôt sauvée par une femme mystérieuse qui lui redonne la vue et la met en lieu sûr. Federico, qui était venu la chercher pour abuser sexuellement d'elle et la tuer, fait la connaissance d'un petit horrible mage, qui lui révèle que la reine est protégée sous le nom de Rosismunda par le duc Octavio. Le mage lui propose de se défaire de la reine en envoyant des lettres au duc accusant Rosismunda d'intelligence avec l'ennemi pour assassiner le duc. Le stratagème marche comme prévu et renvoyée par le duc, Beatriz se trouve encore exposée à la malignité de Federico qui cherche à la violer dans un parage solitaire. Mais la dame mystérieuse réapparaît, qui l'arrache des bras du prince, mettant à la place un lion qui le blesse grièvement. La dame mystérieuse ramène Beatriz dans un hameau où elle rencontre l'Empereur d'Allemagne, sa femme et leur fils de six ans, qui charmés par les grâces de Beatriz, l'emmènent et la nourrissent chez eux. Le mage propose encore à Federico de nuire à Florinda (c'est le nom qu'adopte alors Beatriz) en tuant le petit enfant de l'Empereur pour qu'on inculpe Florinda de ce meurtre. Ce projet diabolique réussit de nouveau et l'Empereur ordonne la décapitation de sa protégée. Mais la dame mystérieuse apparaît encore et enlève Beatriz au moment de l'exécution, à la confusion de tout le monde. Cette fois-ci, elle la conduit dans un bois pour qu'elle vive tranquille en ermite. Peu après, un émissaire de l'Empereur vient arrêter l'exécution car l'enfant avait ressuscité, ce qui a permis d'innocenter Florinda et lui faire justice. Huit ans s'écoulent après lesquels on apprend que la peste ravage la Hongrie et que Federico en est gravement atteint. Alors la dame mystérieuse se présente de nouveau à Beatriz et lui révèle qu'elle est la Vierge Marie et qu'il est temps qu'elle reparte à la cour de son mari, le roi de Hongrie. Ainsi, travestie en «médecin miraculeux» apportant le remède contre la peste que la Vierge lui donne, Beatriz sillonne le royaume pour guérir les gens avec une herbe magique. Mais la guérison ne peut avoir lieu que si le malade confesse tous ses péchés devant la personne que Beatriz aura désignée; en cas contraire, au lieu de guérir, le malade meurt subitement. Le roi fait appeler le médecin 
miraculeux pour guérir son frère, qui face à la peur de mourir, confesse tous les outrages qu'il avait faits à la reine. Ladislao est au désespoir à cause de son aveugle erreur de condamner injustement sa femme, mais comme Beatriz le voit sincèrement repenti, elle se découvre et lui pardonne son injustice. Federico aussi lui demande pardon, tandis que la Vierge fait son apparition et le mage diabolique est puni et réduit en une fumée noire et dense. Malgré tout, Beatriz n'a pas l'intention de rejoindre son mari, car elle préfère rentrer dans un couvent et renoncer au monde. Ses pas sont suivis par Ladislao, qui rentre dans l'ordre de Saint-Benoît. Dans ces circonstances, puisque l'État ne peut pas rester sans un chef, Federico est proclamé roi et on lui accorde pour épouse la sœur de Beatriz, Isabella. Le récit finit donc par un mariage mais avec l'idée et le sentiment essentiels d'avoir été témoins de la conduite exemplaire et magnanime d'une femme exceptionnelle, la victime de l'histoire, une épouse toute de chasteté et de vertu, sachant endurer stoïquement la diffamation, la calomnie, l'humiliation, et toutes les atrocités que l'on ne peut imaginer supporter, mais qui, par sa grandeur d'âme et d'esprit, en est sortie plus glorieuse et triomphale (héroïque, si l'on veut), telle une «persécutée triomphante» (traduction littérale du titre de la nouvelle de María de Zayas.

Sachant que Boisrobert écrit sa nouvelle en 1657, époque où l'exigence de vraisemblance est une condition majeure en France, on peut aisément deviner quels éléments de ce récit il est forcé d'abandonner, non seulement par le caractère extrêmement fantastique - bien que plaisant - de la nouvelle de Zayas, mais aussi par la nécessité de vraisemblance, de bienséance et de concentration par rapport aux unités, devenues règles suprêmes en France. Cela va sans rappeler que la nouvelle française de notre auteur fonctionne à l'instar d'une pièce de théâtre, comme une entité dramatique. Alors, il réduit au maximum le personnel héroïque ainsi que l'intrigue, ne laissant que la principale au détriment des secondaires, qui sont nombreuses chez Zayas. L'abbé se concentre donc sur la naissance de la passion «incestueuse», l'accusation et la condamnation de la reine, ainsi que la révélation de la vérité au roi pour rétablir l'ordre, un ordre que l'on peut tout à fait identifier au traditionnel procédé des reconnaissances et au mariage final (entre Irène et le prince Tindare), à l'exemple de celui que Federico contracte avec la princesse Isabella d'Angleterre.

Voici donc le remaniement opéré par Boisrobert dans son récit: Ladislas, roi de Hongrie, est obligé de quitter le royaume pour soutenir la guerre contre les Turcs. À la tête de son armée, il laisse en qualité de régents Théodore son épouse et Tindare son frère. Celui-ci avait été amoureux d'Irène, cousine de la reine, et l'on croyait qu'il l'était encore. Mais depuis un certain temps, on ne lui voit plus le même enthousiasme. Irène l'aime pourtant passionnément; et la reine qui craignait qu'il ne prenne trop de licences avec sa parente, lui avait défendu de revoir le prince, jusqu'à ce qu'il eût fait preuve de la sincérité de ses feux en la demandant officiellement en mariage. Irène cherche et trouve le moyen d'avoir une explication de Tindare sur sa froideur envers elle, mais elle apprend avec dépit le peu d'amour que le prince lui témoigne désormais. La reine, qui s'est aperçue du chagrin de Tindare et qu'elle attribue à la défense qu'elle lui a faite de voir Irène, l'envoie chercher. Ce prince a une longue conversation avec elle; la reine est surprise de la froideur avec laquelle il répond quand elle lui parle d'Irène, mais il cherche à lui apprendre subtilement que c'est d'elle dont il est réellement amoureux. Un peu de scrupules le retient encore pour se déclarer ouvertement. En effet, il n'aime plus Irène, subjugué plutôt par Théodore, qui croyant que par timidité il n'avait pas voulu lui avouer l'amour qu'il ressentait pour la cousine, sort et va chercher celle-ci. C'est alors qu'un courrier arrive pour annoncer la grande victoire que le roi vient de remporter sur les Turcs. Le prince vient aussitôt en rendre compte à la reine, et tirant parti de cette occasion pour se déclarer, il lui avoue ouvertement l'excès d'amour qu'il éprouve pour elle. La reine lui fait voir avec retenue l'énormité de son «crime», mais cette bonté ne fit qu'enflammer davantage le prince, qui s'évertue à lui baiser la main sensuellement. Alors Théodore lui défend de l'approcher et le quitte sur le champ. En même temps, une 
nouvelle lettre du roi informe Tindare qu'en suite de la victoire (obligé de donner du repos à son armée), il veut profiter de l'occasion pour venir revoir la reine. Il le prie de ne pas l'en avertir pour jouir du plaisir de la surprise. Le prince, ne doutant pas un instant que la reine allait dénoncer son inconduite, la devance et s'en va trouver le roi au château de la Tour Blanche, où il leur avait donné rendez-vous. Tandare fait semblant de paraître devant lui pénétré de la plus vive douleur. Ladislas, qui aimait tendrement son frère, lui en demande la cause. Peu soucieux de son imposture, le jeune homme confie au roi que la reine avait cherché à le séduire et à souiller son lit nuptial. Trop crédule, le roi entre dans la plus grande et aveugle fureur, et ordonne à Ramèse, son confident, d'aller plonger un poignard dans le cœur de son infidèle épouse. Ramèse obéit avec regret, et retourne bientôt apprendre au roi qu'il a exécuté ses ordres cruels. Irène paraît ensuite, qui prouve au roi l'innocence et la vertu de son épouse en montrant une lettre d'amour que le suborneur avait eu l'audace d'adresser à Théodore. Ladislas se livre alors aux plus vifs regrets et veut suivre la reine dans sa mort. Quand celle-ci voit son mari sincèrement repenti et accablé de remords, Théodore, qui l'écoute à l'écart, cachée derrière quelques domestiques, paraît subitement à ses yeux. Le roi enchanté à cette vue se jette à ses genoux et lui demande pardon; il l'obtient sans peine, mais elle lui demande aussi celui de Tindare, à condition qu'il épouse Irène. Ladislas ne rejette pas la demande de sa vertueuse épouse; ainsi, il pardonne à Tindare. Quant à Ramèse, il meurt deux ans après dans la célèbre bataille de Varna, petit détail dont on sent bien la gratuité, tandis qu'on n'apprend pas trop ce que deviennent les rapports entre le roi et sa femme. Ainsi, la portée de ce qui aurait pu être l'image exaltée et magnifiée de Théodore reste édulcorée et de peu de portée.

On perçoit bien que malgré le caractère narratif de l'œuvre, Boisrobert n'est pas moins soucieux de concentrer l'intrigue au maximum à l'instar d'une pièce de théâtre à la française, même si nous avons aussi une action secondaire tenant aux rapports amoureux entre Irène et Tindare, car il évite l'éclatement de l'espace et, dans une moindre mesure, celui du temps, ce dont la technique narrative lui permet de se prévaloir. Mais l'on peut voir ici une grande défaillance. Cette action secondaire, annoncée dès le début (ce qui aurait permis de bien nouer l'intrigue et donner sur un dénouement cohérent et solide) prend cependant trop de place et semble manquer d'efficacité puisque le roi restera roi, la reine aussi, et le prince restera prince. C'est à dire que le renoncement au trône était justifié dans la nouvelle espagnole puisque le repentir du fauteur se traduisait en une sorte de punition pour une décision hâtive qui condamna la reine à une souffrance longue et injuste, mais il n'en va pas de même chez Boisrobert, qui laisse les choses en l'état et personne n'est vraiment puni. Cette action seconde est donc gratuite et d'autant plus inutile que le personnage d'Irène n'a aucun relief.

Ajoutons que l'intrigue, telle que nous l'avons rapportée, est exactement la même que celle de la tragi-comédie Théodore, reine de Hongrie, avec quelques ajustements nécessaires pour faire le passage du mode narratif au mode dramatique. Cet ajustement consiste en l'introduction d'une intrigue amoureuse secondaire entre Irène et Tindare, que l'auteur concentre pratiquement dans le tout premier acte. Boisrobert crée ainsi le personnage de Carinte, la suivante d'Irène, qui permet de remplacer le récit par le dialogue, encore que ce dialogue soit quelque peu artificiel, puisqu'il ressemble à bien d'égards à des monologues, les personnages n'établissant pas de vrais rapports d'échange. Pour éviter que cette situation ne soit trop récurrente, Boisrobert fait aussi intervenir Téralde, le confident de Tindare que l'on retrouve dans la nouvelle. Irène peut ainsi faire part de ses inquiétudes non seulement à Carinte, mais elle les fait aussi partager entre Carinte et Téralde, qui prennent le relais de ses inquiétudes et ouvrent même la pièce. On remarquera la difficulté qu'a éprouvée Boisrobert à dynamiser la communication entre les personnages par la création de nombre de scènes où il est question d'un monologue, comme dans I, 3, 5, 6 ; III, 6, 12 et IV, 4, 7, ainsi que d'autres scènes, comme la scène treize de l'acte II, où même s'il y a deux 
personnages, elles fonctionnent aussi comme de vrais monologues. En effet, Tindare ne s'adresse jamais directement à Téralde; celui-ci sert seulement à donner l'impression qu'on est dans le théâtre, à telle point que ses interventions se comportent comme de vraies didascalies, comme dans le vers 942: «Il se tire à l'écart, \& tout bas il la lit [la lettre $\left.{ }^{28}\right] \gg$. Un vers de ce type, on le voit bien, doit en plus se déclamer en aparté, même si l'auteur ne l'a pas indiqué dans le texte original. Ce sont donc des situations qui pourraient assez prouver que notre abbé a d'abord écrit sa nouvelle et qu'il l'a ensuite adaptée pour le théâtre, mais non l'inverse ${ }^{29}$.

Mais ce qui est vraiment important à retenir dans le cas de L'Inceste supposé de Boisrobert c'est que l'on trouve dans cette nouvelle, et donc dans la Théodore aussi, tout le matériau nécessaire qui aurait permis à l'abbé d'écrire et la nouvelle et la pièce à partir d'une seule et unique source, La perseguida triunfante en l'occurrence. On constate ainsi la présence des mêmes éléments que l'adaptateur a tirés de l'Espagnole: la déclaration de guerre qui oblige le roi à partir, la désignation par le roi de sa femme et de son frère comme régents de Hongrie pendant son absence, la maladie qui ronge Tindare pour l'amour de la reine, les efforts de celle-ci pour dissimuler devant ses avances, la lettre qu'il lui adresse, le fait qu'elle se taise et qu'il interprète mal à propos ce silence, la manière dont il se résout à déclarer ouvertement sa passion, ses écarts de conduite notamment en lui baisant langoureusement sa main, le fait que la reine demande à ses domestiques de ne jamais la quitter pour dissuader le prince de toute audace licencieuse, la lettre qui annonce le retour du roi, le fait que celui-ci fasse savoir qu'il n'est qu'à une «demie journée» de distance de la ville où il pense pouvoir arriver dans la soirée, comment le prince le rejoint et devance la reine pour renverser l'histoire et l'accuser de l'avoir séduit, la réaction insensée et aveugle du roi qui la condamne injustement sans vouloir même s'enquérir de la réalité des accusations, comment il envoie un suppôt pour la faire assassiner, les remords tardifs qu'il éprouve d'avoir pris une décision précipitée, comment elle est épargnée de la mort (par la Vierge chez Zayas et par la lettre d'amour de Tindare qui prouve l'innocence de Théodore chez Boisrobert), comment elle est conduite dans un bois où le meurtre doit avoir lieu, enfin comment le roi apprend l'imposture de son frère, combien il se morfond en remords d'avoir cru à un mensonge et d'avoir fait tuer sa femme, comment on le détrompe de la mort de celle-ci, le fait qu'elle accorde son pardon au suborneur, comment il se repend aussi de ses méfaits, enfin le mariage que l'on cherche à concerter entre le prince et une autre princesse (Isabella, la sœur de la reine chez Zayas; Irène, la cousine de la reine chez Boisrobert). En revanche, Boisrobert consacre tout le dernier acte à faire connaître au roi l'imposture de son frère et montrer combien il souffre d'avoir condamné injustement sa femme. Le Français met ainsi l'accent sur l'aspect amoureux du mari repentant tandis que Zayas focalise sur la méchanceté naturelle des hommes qui cherchent à détruire et à discréditer les femmes. En termes de réception, les contextes socioculturels sont différents. Elle ne laisse qu'une place très réduite au repentir mais comme venant trop tard puisque le mal est déjà fait.

Dans ces circonstances, on comprendra qu'il soit difficile d'attribuer une autre source que celle de La perseguida de María de Zayas à la nouvelle de Boisrobert, et par définition, à sa tragi-comédie Théodore, où il resserre encore plus la scène. Cependant les Remarques de Somaize doivent nous alerter sur ce point, car il s'agit d'un contemporain qui accuse Boisrobert d'avoir pillé un dramaturge qui avait travaillé sur un sujet semblable presque vingt ans plus tôt. En effet, dans sa diatribe contre Boisrobert et sa pièce, Somaize cite quelques vers de la tragi-comédie L'Inceste supposé de La Caze que notre abbé aurait «volés» à cette pièce. C'est ainsi qu'il l'affirme après avoir consulté l'œuvre de La Caze:

Toutesfois comme la presence des objets r'allument les passions \& reveillent l'esprit, ma memoire qui conservoit cherement l'idée de ces belles expressions, me fist souvenir de l'inceste du sieur de la Caze, où je les avois veuës; mais comme je ne porte pas 
aisement un jugement de cette nature, j'ay voulu avant d'en parler relire l'ouvrage de cét homme, où je les ay trouvez dans la Scene cinquiéme du second Acte page 55. En verité cette conformité de pensées, d'expressions, de paroles, \& d'occasion, est admirable, ou plustost ce vol, car enfin qui vit jamais deux hommes tomber si directement dans un mesme sens, que de ne se differer pas d'un seul mot l'un de l'autre: je proteste que si l'inceste supposé n'avoit esté fait plus de dix-sept ans devant cette Theodore, j'aurois accusé son Autheur d'injustice. (1. 1092-1102)

Voici les vers en question; dans la colonne de gauche, ceux de La Caze que cite Somaize (et qui se trouvent aux pages 53 et 54 de l'édition de Paris, chez Toussaint Quinet, 1640), et dans la colonne de droite ceux de Boisrobert que nous rapportons pour les comparer (acte IV, scène 4, vers 1162-1190):

\section{CARISMOND}

Vain \& coupable objet de ma juste fureur,

Tout ensemble du jour la merveille \& l'horreur,

Ton chastiment de prés suivra ton insolence,

Mon amour en fureur change sa violence,

Et tout ce qu'il medite en ce funeste ennuy,

C'est de te perdre enfin, $\&$ te perdre avec luy;

C'est d'enfermer ton nom dedans ta sepulture,

Et de le rendre horrible à la race future :

Le perdre, ah! mon amour revoque cét arrest!

Cét objet est charmant tout criminel qu'il est:

La noirceur de son crime a ma gloire blessée;

Mais elle n'oste pas ses traits de ma pensée,

Il est aimable \& traistre, il est doux \& cruel;

Sa beauté jette un feu, son crime un coup mortel:

Vains attraits, vain amour, sortez de ma memoire,

Si l'un blesse mon nom, l'autre blesse ma gloire;

Et d'autant que la gloire est le plus digne objet,

Perdons pour la vanger l'amour \& son sujet.

Tonne, Ciel, fais pleuvoir un deluge de flame,
Et des feux de ta foudre esteins ceux de son ame, Ou mon bras animé par un juste courroux, Si vous ne me vengez me vengera sans vous ; L'impudique en mourra, je le veux, je le jure, Ma fureur dans son sang lavera mon injure Si vous estes encore ennemis du forfait, Dieux, j'en fais le dessein, approuvez-en l'effet.

\section{LADISLAS}

QUOY je la reverrois dans ce desordre extresme, Je t'abuse, mon frere, \& m'abuse moy-mesme, Non ses attraits sont morts, je ne les veux plus voir,

Ses beaux yeux quoy qu'en pleurs n'auroient plus de pouvoir,

Je verrois qu'ils auroient noyé dedans ces larmes, Sa vertu qui faisoit le plus beau de ses charmes.

Cette vertu faisoit l'ornement de ma Cour,

Elle fondoit ma gloire autant que mon Amour,

Cette vertu rendoit ma flâme legitime,

Et je n'ay plus d'Amour où je n'ay plus d'estime,

J'abhorre en cet instant que je voy mon erreur,

Des charmes adorez qui me feroient horreur,

Et je vay par un coup \& tragique \& funeste,

Montrer à l'univers combien je les deteste,

Theodore est infâme, elle ne m'est plus rien,

Mais comme nostre honneur se confond dans le sien,

Il faut que par sa mort j'expie un Adultere,

Qui n'est que dans l'esprit, qui n'est qu'imaginaire,

Mais je ne puis souffrir un soupçon seulement,

Dans ce cœur delicat blessé mortellement ;

Beauté rare \& divine autrefois adorée,

Pourquoy pour mon malheur t'es-tu deshonorée?

Et pourquoy te perdant, \& t'oubliant ainsi,

Faisant ton deshonneur, fais-tu le mien aussi,

Crois-tu que sans honneur Ladislas puisse vivre? 
Ce mouvement est juste, enfin il le faut suivre,

De ton déreglement tu recevras le prix,

Tu mourras, tu mourras, le conseil en est pris, Perfide.

On s'aperçoit que si l'esprit de la tirade en est le même, on ne peut que constater que Somaize va trop loin dans ses accusations, car à moins que Boisrobert n'ait modifié ses vers après avoir lu les Remarques de son détracteur, on ne voit pas en quoi ils «ne differe[nt] pas d'un seul mot l'un de l'autre». Chez La Caze, le Carismond balance et se trouve devant un vrai débat «cornélien», tandis que chez Boisrobert nous avons un Ladislas bien décidé à laver son honneur en assassinant la reine. Rappelons que cette critique parut le 6 août 1657, tandis que la pièce de théâtre fut jouée le 15 juin à l'Hôtel de Bourgogne et que le texte ne fut achevé d'imprimer que le 15 novembre de la même année (bien que la page de titre affiche la date de 1658 , qui devrait être une fausse date $^{30}$ ). Cela explique aussi que dans la dédicace de la pièce à la Procureuse Générale, Mme Foucquet, Boisrobert nous fasse savoir que:

Si ma Theodore qui a esté accusée fort injustement n'avoit esté pleinement justifiée, je me serois bien gardé de vous demander pour elle l'honneur de vostre protection, quoy qu'elle en ait besoin dans un siecle où je voy si peu d'indulgence $\&$ de justice. (1. 1-3).

$\mathrm{Si}$ les Remarques parurent après la création de la pièce, on pourrait aisément imaginer que Boisrobert soit rapidement venu corriger le texte et remanier la version écrite de l'œuvre, surpris par les accusations de Somaize. L'œuvre créée peut dont bien différer de l'œuvre imprimée, mais il n'y a pas de témoignages écrits découverts à ce jour qui le prouvent. Trois mois donc, avant la publication de sa pièce, auraient suffi à l'abbé pour introduire les changements nécessaires, car autrement on ne comprendrait pas que son détracteur soit si précis dans les vers qu'il rapporte avoir été pillés, et que pourtant on ne trouve pas (ou plus) dans la Théodore. Cela permet aussi de comprendre pourquoi l'abbé parle dans la dédicace des accusations injustes qu'il a subies. Il est vrai aussi enfin que Somaize est aux aguets sur tout ce qui se passe sur la scène et dans les ruelles, désireux de se faire valoir et remarquer. L'occasion aurait été trop bonne pour s'en prendre à Boisrobert, qui y jouit d'un certain renom, et qui paraît ainsi une proie facile qui manifestement ne se doutait qu'une telle diatribe aurait pu lui subvenir.

Mais Andras Klein vient à affirmer «[qu]'il est donc possible qu'il y ait eu une édition antérieure à celle que nous connaissons aujourd'hui, puisque Somaize devait connaître le texte avant la parution de la dédicace, le 15 novembre $1657^{31_{1}}$. Cependant, outre qu'il n'y pas de raison pour qu'il existe un imprimé antérieur à celui de l'édition que nous rapportons ici et à laquelle A. Klein fait référence, c'est également négliger le fait qu'à cette époque ce n'était vraiment pas nécessaire qu'un texte passe par une imprimerie pour être connu, sans parler des délais qu'on vient d'évoquer et qui permettaient aisément à Boisrobert de remanier sa tragi-comédie. En effet, dans la culture littéraire française du milieu du XVII ${ }^{\mathrm{e}}$ siècle, les textes pouvaient facilement circuler sous forme de manuscrit, notamment dans les ruelles où les poèmes dramatiques étaient «éprouvés» et préalablement soumis au public le plus choisi de la société galante pour voir s'ils étaient susceptibles de plaire. Cette pratique est d'autant plus courante que de l'abbé de Pure s'en fait l'écho tout au long de son roman La Prétieuse, où il nous explique qu'il s'agit là de l'une des plus importantes occupations des dames :

L'objet principal, et qui occupe tous leurs soins, c'est la recherche des bons mots et des expressions extraordinaires; c'est à juger des beaux discours, et des beaux ouvrages, pour conserver dans l'Empire des Conversations un juste temperament entre le stile rampant et le pompeux. Elles se donnent encor charitablement la peine de censurer les mauvais Vers, et de corriger les passables; de travailler les dons de l'esprit, et les mettre si bien en œuvre, qu'ils puissent arrester le sens, élever le commerce de leurs plaisirs, et les rendre aussi spirituels que sensibles ${ }^{32}$.

Nous savons que Boisrobert était «un docte en droict de Chevet», le «directeur du 
royaume de Coquetterie» et le «grand prestre des ruelles», comme l'appelle Somaize lui-même. C'était aussi son habitude de faire lire ses pièces dans les «ronds» galants avant de les donner au public, comme il le signale dans la dédicace de la Cassandre à l'archevêque de Nemours, qui se trouvait à ce moment-là chez Marie d'Orléans, «l'admirable Nitocris»:

Si vous vous souvenez que ce fut en vostre presence, qu'une grande Princesse souffrit avec plaisir la premiere lecture qui a esté faite de cette TrageComedie, vous vous souviendrez aussi des advis obligeans qui me furent donnez par Vous \& par Elle, pour en augmenter les agrémens; $\&$ ainsi vous n'aurez pas esté fort surpris de la grande reputation qu'elle s'est acquise. (1. 1-4)

L'obscur Somaize, qui ne devait pas être aussi jeune qu'on pourrait le penser, vu qu'il «se rappelle» les vers de La Caze, ne révèle pourtant son existence, jusqu'alors inconnue, qu'à l'occasion de son acerbe diatribe contre Boisrobert; c'était un émule de notre abbé, et sans aucun doute cherchait-il à se faire remarquer dans cette société où sa victime trônait en maître. On ignore la suite de l'affaire, Boisrobert étant déjà très âgé pour répliquer à des attaques si rudes et gratuites venant d'un inconnu ${ }^{33}$, mais cette tactique d'attaque de Somaize n'est que la première d'une longue liste par rapport à laquelle Molière ne tardera pas à en faire les frais aussi, notamment à l'occasion de la représentation des Précieuses ridicules en 1659. Ce qui semble certain c'est que Somaize s'était introduit dans le même monde que Boisrobert, ou du moins l'approchait-il, et c'est sans doute dans la ruelle d'une des femmes galantes qui figurent dans son fameux Dictionnaire, qu'il a dû entendre les vers de la Théodore. Aussi devait-il avoir une copie manuscrite de la pièce car il cite d'autres vers dans ses Remarques que l'on ne trouve plus dans la pièce de Boisrobert, et qui devaient figurer juste après le vers 1499 de notre édition de cette tragi-comédie, à savoir:

Il voit vostre douleur \& touché de vos larmes,

Il vous rendra la Reine avecque tous ses charmes,
Si son œil peut encor en revoyant le jour,

Voir ce grand repentir meslé de tant d'amour ${ }^{34}$.

On peut noter aussi que le vers de la ligne 1267 est modifié: «Pourra faire, Seigneur, ce miracle important », tandis qu'on lit dans la pièce de Boisrobert: «Pourra faire à vos yeux ce miracle important» (v. 1499). Et plus loin, aux lignes 1284-1285 et 1287-1288, Somaize cite quatre autres vers qui ne sont plus dans l'édition Lamy et qui devraient paraître à cet endroit, à savoir, deux déclamés par le roi :

Et pourquoy me voyant languir dans cette attente,

Ne soulageois-tu pas mon ame impatiente?

Et la réponse que lui fait Ramese:

J'apprehendois, Seigneur, qu'apres un si grand mal,

Un grand bien impreveu ne vous devint fatal.

Mais l'inexistence de ces vers dans l'imprimé original de sa pièce doit obéir à des raisons totalement différentes à celles de pilleur qu'on lui attribue, car aucun de ces vers n'a d'équivalent dans la pièce de $\mathrm{La}$ Caze. Auquel cas c'est Somaize qui devrait donc être accusé de diffamation. Mais ses accusations sont-elles entièrement fausses? On ne peut pas nier qu'en lisant la pièce de La Caze, il y a des ressemblances avec la Théodore de Boisrobert, et seulement avec la tragi-comédie, non avec la nouvelle, du point de vue de la structure. Il est donc possible que pour la composition de sa pièce, notre abbé ait pu consulter L'Inceste supposé de son prédécesseur à titre de référence ou de modèle d'organisation. Car qu'en est-il de la pièce de La Caze vraiment? Voyons d'abord l'intrigue.

Carismond, roi de Hongrie, se trouve sur le front de bataille dans la guerre contre les Turcs, tandis qu'à la cour sa femme, la reine Alcinée, et son frère Clarimène, gouvernent le royaume en son absence. Le prince est passionnément amoureux d'Alcinée, qu'il tente de séduire. La princesse Clorinie, qui est amoureuse du prince et qui en était aimée, lui reproche son soudain 
délaissement. Mais il répond qu'il éprouve en ce moment une profonde passion amoureuse, ce qui induit Clorinie à croire qu'il parle d'elle. Près du château d'Albe, où le roi est rentré en secret après avoir remporté une bataille contre l'ennemi, il avoue à Philon, son confident, qu'il ne résistait plus au désir de revoir sa femme. $\mathrm{Au}$ palais, Clarimène prend courage et déclare ouvertement sa flamme amoureuse à la reine, dont la colère ne se laisse pas attendre, le menaçant même de dénoncer ses écarts auprès du roi. Clorinie et sa suivante Mélistée, qui n'étaient pas loin, entendent la conversation et apprennent choquées la passion déréglée du prince. Éconduit et humilié par la reine, celui-ci songe à se venger en racontant au roi une version déformée de l'histoire. L'occasion se présente lorsque Sidion, son confident, vient l'informer que le roi est tout près de la ville. Clarimène le rejoint aussitôt et lui dit que la reine a tenté de le séduire. Trop crédule, Carismond ordonne à Philon de tuer sa femme dans un bois éloigné et de lui en apporter le cœur, pour prouver qu'il a bien accompli sa mission. Sidion, qui en fait est un espion de Clorinie, rapporte à celle-ci le menu détail de la cruelle décision du roi. La princesse, qui est venue dans le bois empêcher le crime, montre à Philon l'innocence de la reine. Mais celui-ci ne peut la laisser vivre sans que le roi ne le tue à son tour. Alors ils s'accordent pour masquer le crime et rapporter au roi le cœur d'une biche. Lorsque Clarimène apprend le meurtre de la reine, il tente, désespéré, de se suicider, puis s'en va dans la forêt chercher la dépouille mortelle. Il y retrouve Alcinée, toute de blanc vêtue, ce qui lui fait penser qu'il s'agit d'un spectre. Elle lui dit que son âme est prête à lui pardonner à condition d'avouer son crime au roi. C'est ce que le prince ne tarde pas à faire. Philon vient apporter au roi «le cœur d'Alcinée», que celui-ci montre à son frère, dont le chagrin le conduit à avouer au roi ses calomnies contre la reine. Repentant et furieux, il ordonne l'emprisonnement du prince et la construction d'un beau cercueil pour sa femme. Philon et Clorinie persuadent celle-ci de rentrer dans le cercueil et remplacer l'effigie que Carismond avait commandée en son honneur, pour qu'elle puisse entendre ses plaintes et son repentir. Alors persuadée de la sincérité de son mari, après avoir effectivement entendu le chagrin qui le ronge, la reine sort de son cercueil et lui pardonne. Clarimène vient aussi, qui se montre toujours subjugué par la reine, mais il lui demande pardon. Elle le lui accorde à condition d'épouser Clorinie. Le roi clame vengeance mais la reine obtient qu'il envisage au moins son pardon.

Il ne peut y avoir aucun doute que nous avons affaire à une autre version de la même histoire. On retrouve chez Boisrobert le même personnel dramatique de l'intrigue secondaire ainsi que l'équivalent de certaines scènes où il est question du repentir du roi et de son frère, après l'accusation et le meurtre supposé de la reine, même si dans la nouvelle de María de Zayas les événements se déroulent dans le même ordre chronologique. On ne peut donc pas exclure l'hypothèse que Boisrobert ait composé sa pièce en ayant celle de La Caze sous les yeux. Mais comment se peut-il que l'intrigue de la pièce de La Caze soit aussi proche de celle de Boisrobert et qui plus est de la nouvelle de María de Zayas, qui à la base est postérieure à l'œuvre de La Caze? Existe-t-il la possibilité que l'œuvre de l'Espagnole soit mal datée et qu'elle soit parue avant la pièce de La Caze, ce qui pourrait enfin résoudre heureusement ce réseau confus d'influences? Nous manquons d'élément pour cela faire.

Mais le deuxième constat que l'on fait avec surprise c'est la ressemblance de la tragicomédie de ce dernier avec la nouvelle de l'Espagnole, qui par définition ne pouvait être connue du Français. Faut-il donc croire que l'écrivaine n'était pas la seule détentrice de ce qui pourrait être une légende qui devait être connue partout en Europe? A. Klein rappelle qu'Alexandre Hardy a composé en 1618 une pièce portant le même titre de L'Inceste supposé, œuvre aujourd'hui disparue, mais qui figure bien dans le Mémoire de Mahelot comme appartenant à cet auteur ${ }^{35}$. La Caze s'en était-il inspiré? C'est possible, autrement le phénomène serait inexplicable, à cause de quelques aspects de décor du dernier acte notamment, que Mahelot a consignés: 
L'Inceste supposé Piece de Mr Hardy. Il faut au Milieu du theatre, une chambre funebre, a un des costez ou il y ayt une piramide Pleine de bourgies et un cœur dessuz, le tout tendu de Noir, avec des larmes, a un des costez un hermitage, ou l'on monte et descend, ladicte chambre souvre et ferme au cinquiesme Acte, il faut aussy des dards et javelotes.

En effet, il n'est jamais question d'un cœur ni d'une chambre funèbre chez María de Zayas ni chez Boisrobert, alors qu'on retrouve ces deux éléments dans la pièce de La Caze. Seul Hardy aurait pu, a priori, lui suggérer ces aspects. Nous manquons d'éléments pour remonter à l'origine de cette question de grand intérêt, mais il semblerait, suivant A. Klein, qu'une légende du Moyen Âge, contenant le motif de la reine injustement accusée et condamnée pour adultère avec le frère du mari absent, soit présente dans la culture populaire suédoise, irlandaise, néerlandaise, toscane, croate, roumaine et polonaise. En Italie, elle serait connue sous le nom de L'Impératrice de Rome ${ }^{36}$. L'hypothèse ne paraît pas sans fondement quand on constate avec intérêt qu'on trouve dans L'Heptaméron (1558) de la reine Marguerite de Navarre une nouvelle, la seconde, où il est question d'une histoire très similaire, mais où les personnages sont de bas rang, à savoir des villageois. Cela pourrait ainsi confirmer l'hypothèse de Klein ${ }^{37}$. Enfin, il existe aussi la possibilité que des dramaturges espagnols, comme Lope de Vega, qui avait déjà travaillé à ce sujet, aient pu servir d'inspiration à Hardy et à María de Zayas à la fois ${ }^{38}$, mais Lope de Vega ne serait dans ce cas-là qu'un maillot dans la longue chaîne de versions d'une légende célèbre. Il est vrai aussi que la version romancée de María de Zayas se prête à de plus longs développements avec un luxe de détails et de rallonges qui servent à mettre en valeur l'innocence de la reine et sa vertu exemplaire souillées par la perversité des hommes. C'est en ce sens que l'on peut établir que La Caze d'abord et María de Zayas ensuite n'ont de rapport que ce que la tradition établit à travers un récit légendaire qui circule un peu partout en Europe, ce que confirmerait la reprise d'un motif par des auteurs qui n'ont de lien apparent entre eux. Mais pour faire justice à Boisrobert, il est certain qu'il reste beaucoup plus près du modèle que lui offre la nouvelliste espagnole que de la pièce de La Caze, à cause de la série de détails que nous avons énumérée plus haut et qui semblent provenir de source hispanique, même si le schéma structural de la pièce de son prédécesseur y est pour quelque chose.

\section{Plus d'effets que de paroles}

Le débat sur les sources de cette nouvelle est beaucoup moins âpre que par rapport aux cas précédents, car la critique est assez consensuelle en ce sens que Boisrobert a eu recours à la pièce dramatique de Tirso de Molina Palabras $y$ plumas, parue pour la première fois dans le recueil de Doce comedias nuevas del maestro Tirso de Molina. (Primera parte de la comedias), en 1627. Losada Goya figure parmi les derniers critiques en date à se faire écho de ce fait ${ }^{39}$, qui le confirme comme étant généralement admis. Mais Scarron lui aussi avait fait une adaptation de cette œuvre bien avant Boisrobert. Il s'agit d'une nouvelle insérée dans le recueil des Nouvelles tragi-comiques, parues en 1655 (privilège de 23 avril et achevé du 26 octobre), intitulée aussi Plus d'effets que de paroles (c'est la quatrième en ordre successif) ce qui montre que si elles ont tardé à tomber dans les mains du public, l'auteur pouvait bien travailler sur elles depuis l'année précédente. Elles ont fait d'ailleurs l'objet d'une édition critique préparée par Roger Guichemerre ${ }^{40}$.

Ce critique signale qu'on a proposé diverses sources pour cette nouvelle, comme $L a$ resistencia premiada d'Ágreda y Vargas, où le personnage Juan d'Aragón fait preuve de fidélité malgré les dédains de la belle Gracia, qui finit par l'épouser après la mort de son mari; une nouvelle de Boccace où le héros Federigo est capable de se ruiner totalement pour la femme qu'il aime; ou encore la comedia Amar, servir y esperar de Lope, basée sur une nouvelle de Solórzano, El socorro en el peligro, où Feliciano sauve Dorotea dans toutes les circonstances périlleuses où elle se trouve avant de se voir récompensé pour 
son dévouement. Cependant, tous ces motifs se trouvent bien présents dans la pièce Palabras y plumas de Tirso, qui a donc tous les ingrédients nécessaires pour que Boisrobert comme Scarron y puisent leurs œuvres respectives.

Guichemerre rappelle également que dans son adaptation: «Boisrobert se permet aussi quelques libertés par rapport à son modèle. Il nous informe d'emblée des amours du roi Alfonse et de Sirène, décrit en détail la course de bague, rend Roger responsable du naufrage comme de l'incendie. Dans sa nouvelle, c'est Sirène qui, par l'indiscrétion des valets, apprend que Roger est l'auteur de ces attentats criminels et le révèle au roi; ce sont aussi ses gens qui surprennent chez Roger un envoyé du roi Charles VIII, avec une lettre probant sa trahison. Ailleurs, il suit assez fidèlement l'intrigue de Tirso, conservant notamment la visite de Matilde au roi et le refus de Federic (Próspero) de l'assister dans sa disgrâce. Mais sa nouvelle «hérö̈que» est d'un ton uniformément sérieux [...] et n'admet ni les plaisanteries du gracioso espagnol, ni la désinvolture ou les commentaires satiriques de Scarron ${ }^{41_{\text {}}}$. Guichemerre aborde aussi la question notamment concernant l'adaptation faite par Scarron, qui se caractérise par le ton burlesque que cet auteur adopte, fidèle à sa manière particulière d'écrire, et les libertés plus grandes qu'il prend dans sa version de la comedia espagnole de Tirso ${ }^{42}$.

Pour sa part, la nouvelle de Boisrobert a fait l'objet d'une réédition par Esperanza Cobos ${ }^{43}$ qu'elle accompagne d'une étude succincte qui se fait écho de la brève analyse proposée par Guichemerre dans son édition critique, qui a son tour reprend les analyses faites par Georges Hainsworth. C'est donc à celui-ci que l'on doit vraiment un progrès dans la connaissance des sources de cette nouvelle, tant par rapport à celle de Scarron qu'à celle de Boisrobert.

Enfin, l'érudit G. Hainsworth, qui nous avait dit dans son ouvrage Les Novelas exemplares de Cervantès en France au XVII siècle $^{44}$ qu'on ignorait les sources de cette nouvelle de Boisrobert, vient plus tard nous indiquer qu'il les a trouvées après une recherche exhaustive de la question ${ }^{45}$. Il affirme catégoriquement que c'est à Tirso que remonte le travail de notre abbé ainsi qu'à celui de Scarron. Cette œuvre est romanesque à souhait. La scène se situe à Naples vers la fin du $X V^{e}$ siècle, et décrit deux types très différents d'amants: Próspero de Taranto, frêle et infatué prince italien, inconstant et avare, et don Íñigo de Ávalos, magnanime cavalier espagnol. Rivaux, ils veulent s'attirer l'amour de Matilde de Sanseverino, princesse de Salerno, qui à la fin parvient à se libérer de l'ascendant que le premier a sur elle pour terminer par épouser le second après maintes épreuves d'amour constant et inconditionnel. Une seconde intrigue se greffe à celle-ci qui consiste en la rivalité de Rugero, comte de Celano et cousin de Matilde, qui cherche à la détrôner pour occuper sa place en ayant recours à une série de pièges et de calomnies de trahison. Voici les éléments principaux de la pièce que dégage $\mathrm{G}$. Hainsworth: des scènes romanesques, tant dans la pièce que dans les versions narrativisées, où l'on voit la mesquinerie du prince et la grandeur chevaleresque de l'Espagnol: 1. il organise un juego de cañas en suite d'un procès que Matilde remporte avec succès sur Rugero pour fêter l'occasion; il lui sauve la vie, d'abord quand elle risque de se noyer dans la mer et ensuite lorsque Rugero incendie la maison de l'Espagnol où Matilde est en convalescence; il vend jusqu'au dernier de ses biens pour pouvoir assister et nourrir la princesse; et il offre son arme au messager qui vient dire que le roi a innocenté Matilde, rentrée à Naples. 2. des situations à caractère fortement dramatique, comme les querelles entre Próspero et Matilde (I, 1 et 14, et II, 3) et avec Íñigo (I, 6). 3. des scènes comiques où Gallardo, valet de celui-ci, se moque de son attitude «d'amoureux transi», ou s'inquiète de leur pénurie économique (II, 5, 7, 13-17; III, $8-16)$; 4. des scènes amoureuses où le roi courtise Sirena, sœur d'Íñigo (II, 11 et III, 2): dans la première, il la retrouve chez Laura, sœur de Rugero, où il était venu pour annoncer à celle-ci son désir d'épouser Próspero, mais il est distrait par la beauté de Sirena au point qu'il oublie le motif de sa visite. Mais par rapport à la longue étude comparative qu'il fait sur la nouvelle de Scarron, G. Hainsworth conclut que: «the humour 
and realism of Scarron's version distinguish it radically from that of Boisrobert. The latter maintains a uniform tone of artificial gallantry and, as may be guessed, even suppresses the role of the valet. Boisrobert is no doubt the more "classic" of the two. But Scarron, with his taste for the particular, in psychology and exterior, his cynicism and manifold irony, seems to us incomparably richer than Boisrobert in promise for the future ${ }^{46} \gg$.

Mais que se passe-t-il dans la pièce de Tirso: dans la première journée, on fait connaissance avec Íñigo, noble jeune espagnol, amoureux transi de la princesse Matilde, qui n'a que froideur et mépris pour lui et qui est éprise en revanche de Próspero, prince de Tarente, à qui son père l'a promise. Mais Matilde subit une série de rudes épreuves qui permettent à Íñigo de se montrer amant constant, libéral et dévoué inconditionnel, dans une attitude tout à fait chevaleresque qui contraste de manière frappante avec la lâcheté, la mesquinerie et l'hypocrisie de son amant le prince. Lorsque le comte Rugero, cousin de Matilde, tente de s'approprier de sa principauté, Íñigo parvient à lui trouver des appuis pour favoriser la cause de Matilde, tandis que Próspero reste à distance et ne lui est d'aucun secours. Ensuite, au cours d'une promenade en vaisseau dans la mer, la princesse fait naufrage mais Íñigo la sauve de la noyade, tandis que l'Italien ne songe même pas à mouiller les plumes de son chapeau. Enfin, elle a failli périr dans un incendie dans la maison de l'Espagnol où elle fait sa convalescence, incendie qui a été provoqué par le rival de la princesse, son cousin Rugero. C'est encore à Íñigo que revient l'honneur d'éviter le pire alors que le prince ne songe qu'à se sauver d'abord luimême. Dans la deuxième journée, Matilde est au comble de ses infortunes. Calomniée par Rugero, qui l'accuse d'être en intelligence avec le duc d'Anjou, ennemi mortel du roi de Naples avec qui les rapports sont conflictuels, elle est contrainte de s'exiler et est lâchée par Próspero, qui se tourne même vers la sœur du favori du roi, alors qu'Íñigo refuse de l'abandonner et la met à l'abri chez l'un de ses parents. Une seconde intrigue est ici greffée où l'on voit le roi s'éprendre de la sœur de don Íñigo, Sirena, que Laura, sœur de Rugero, accueille chez elle, et où le roi s'était rendu pour lui montrer son désir qu'elle épouse Próspero lui-même. Íñigo refuse les offres du roi qui veut le nommer général des armées, étant donné ces grandes qualités de général. Il veut lui donner Laura mais l'Espagnol repart avec Matilde. Dans la troisième journée, le roi découvre la trahison et les impostures de Rugero, qui par un quiproquo se met en évidence croyant parler à un complice alors qu'il s'adresse au roi en personne. Rugero est arrêté sur le champ et Matilde peut rentrer à Naples. Elle arrive avec Próspero et prie don Íñigo d'assister à son mariage, ce qu'il déplore par l'ingratitude que cela montre. Le roi veut donner Matilde au prince, mais elle lui rend ses plumes et se détermine à épouser don Íñigo pour récompenser ainsi tant de dévouement, tandis que le roi épouse Sirena, et donne Laura à Próspero.

S'agissant de l'adaptation faite par Boisrobert, qui est postérieure à celle de Scarron et à laquelle on serait tenté de croire qu'il a eu recours, on peut dire cependant qu'il a voulu marquer ses distances par rapport à son rival, dont -comme on peut bien l'imaginer-il repousse le style burlesque et le tour comique qu'il donne à sa production. Boisrobert adopte un ton beaucoup plus grave et sérieux, et élimine le comique que l'on pouvait rencontrer chez Tirso, ce en quoi il s'harmonise et reste homogène par rapport aux autres nouvelles du recueil. Par ailleurs, on ne peut laisser de constater que notre auteur suit de très près la source: même schéma structurel et mêmes données de l'histoire espagnole, comme l'a bien remarqué J.-M. Losada Goya sans pour autant approfondir ${ }^{47}$. Boisrobert a cherché seulement à camoufler la pauvreté et l'indigence dans laquelle sont tombés les héros de la pièce de Tirso en suite de la déflagration de la maison de don Íñigo et de la confiscation des biens par le roi. Notre auteur a dû trouver que ces éléments pèchent contre la bienséance à la française, s'agissant d'une grande princesse et d'un gentilhomme de la plus haute naissance qui méritent un certain décorum et plus de dignité, bien qu'on ne sache pas comment nos héros réussissent à conserver 
leur lustre au milieu de leur misère. Ainsi, alors que chez Tirso l'Espagnol est dans la déchéance la plus totale, chez Boisrobert on lit ceci: «Allons donc, Madame, dans la Barque que je vous ay fait preparer, j'ay de l'argent \& des bagues, rien ne vous manquera pour vostre voyage $\&$ quand nous serons chez le Prince de Caserte nous adviserons à loisir à ce que sera le plus expedient pour vostre salut» (1. 1076-1079). Mais ce n'est là qu'un détail: cela va de soi. En revanche, il a dû trouver absolument sordide l'épisode où le valet Gallardo est obligé de vendre des cure-dents pour pouvoir subsister tandis que le maître est contraint d'aller pécher et de chasser pour nourrir Matilde. Il est certain que pour rester dans une tonalité héroïque et donner cohérence à son récit, la présence d'un valet ayant ce genre de discours et accomplissant des actions si basses contrastait beaucoup avec la finalité et la poétique du genre à la française.

De même, on perçoit bien à la lecture de cette nouvelle, que Boisrobert cherche à mettre en relief l'image de la femme victime de l'infortune et des calomnies des hommes. Notre abbé se montre ainsi fidèle à la tonalité tragique que l'on retrouve aussi dans sa Théodore, Les Coups d'amour et de fortune ou L'Inceste supposé. La femme devient chez lui une malheureuse martyre qui souffre l'infamie et l'injustice, et qui déplore la hâte avec laquelle les hommes la jugent. On le voit à des passages comme celui-ci: «Elle receut donc cet advis au mesme temps que Roger estoit allé rendre compte au Roy de sa commission, \& se resolut aussi-tost par l'advis de Don Cesar \& de Syrene, de s'aller jetter aux pieds du Roy pour se plaindre de l'injustice de cette persecution, \& pour luy faire voir son innocence» (1. 889-892). Et il a des endroits où l'auteur met dans la bouche de la princesse des propos qu'on croirait déjà avoir entendu à Théodore, la reine de Hongrie:

Aussi-tost que Matilde eut jetté les yeux sur cette lettre contrefaite, elle les tourna vers Don Roger qu'elle vit là present, \& luy demanda s'il estoit \& l'accusateur \& le faussaire; comme il ne respondoit rien: Ah ! Sire, luy dit-elle, voyez le visage de Roger $\&$ voyez le mien, il palit le meschant, il se trouble, $\&$ cette émotion qu'il produit contre luy-mesme vous fait voir assez clairement qui de nous deux est criminel; si c'est luy qui m'accuse \& qui a supposé cet escrit comme il y a grande apparence, comment vous laissez-vous persuader par un homme que vous sçavez estre mon plus cruel ennemy, \& qui donneroit toutes choses pour me perdre \& pour se rendre maistre d'un bien qu'il m'avoit injustement disputé? si ce n'est pas luy qui vous a donné ce papier, par lequel vous me jugez criminelle, Où l'avez-vous pris? comme c'est un tesmoin qui ne peut parler, souffrez que je sçache qui le produit contre moy, si vous voulez que je me deffende; car enfin, vous estes trop juste pour me condamner sans m'ouyr, \& pour m'interdire les moyens qu'on laisse mesme à ceux qui sont convaincus de crime; comme je suis fort asseurée de mon innocence, je ne vous demande point de grace, traittez-moy, Sire, avec la derniere severité; mais escoutez-moy, puisque vous avez bien escouté le Traistre qui a suborné la plume avec laquelle on a falsifié cet escrit. Si personne ne parle \& si vous voulez estre seul, \& mon juge \& ma partie, souffrez que j'appelle de vous à vousmesme, ou si vous ne voulez pas connoistre de mon innocence, comme vous avez bien voulu connoistre de mon crime pretendu, je vous demande au moins des Commissaires pour l'examiner, puisqu'il s'agit de l'honneur \& du bien d'une personne aussi considerable que je la suis dans vos Estats, \& qui certainement vous a tousjours esté fort fidelle.

Le Roy qui estoit le plus defiant de tous les Princes du monde \& qui sentoit les approches du Roy de France, dont il sçavoit que Matilde estoit Alliée, s'estoit si fort laissé prevenir par l'accusation de Roger, sans considerer qu'il estoit son ennemy, qu'il ne luy voulut pas laisser le temps de luy parler davantage. Allez, luy dit-il, Madame je sçay vos intelligences \& vos pratiques, sortez promptement de mes Estats, vous avez des Amis icy à qui je permets de vous deffendre, si vous estes innocente on vous fera justice; mais je ne vous puis souffrir icy plus long-temps, choisissez quelque retraitte hors les lieux où vous avez de l'authorité \& qui ne me soit point suspecte.» (1. 932-963).

C'est ce genre de propos qui sont absents de l'œuvre de Tirso, et dont Boisrobert tient à développer longuement l'esprit pour entretenir ce sentiment d'injustice qui le hante tant depuis la disgrâce qu'il a subi auprès du cardinal Richelieu, lorsqu'il était son secrétaire littéraire. C'est un thème récurrent qui revient dans sa prose et dans ses tragi-comédies.

À chaque fois, on est choqué, indigné, étonné et outré chez Boisrobert par la manière effrontée dont les antihéros se dégagent de leurs devoirs de gentilshommes, comme le 
prince Fédéric (lors du naufrage, de l'incendie et du procès contre Matilde) ainsi que Tindare (qui calomnie Théodore auprès du roi). C'est finalement une autre femme (ici Sirène) qui porte secours à la femme victime d'injustice (comme la Vierge a fait avec Théodore), les seules personnes à qui on puisse faire vraiment confiance parce que désintéressées et compréhensives dans les moments de détresse.

Enfin, quant au dénouement, Boisrobert amplifie la découverte de la trahison de Roger et insiste sur l'aveuglement choquant du roi devant tant d'évidences de fidélité et de dévouement montrées par César et Matilde. Celle-ci nous est ainsi présentée vers la fin comme une sainte, incapable de méchanceté et toute faite de pardon envers ses ennemis déclarés: «Quoy que Roger fust extresmement coupable, Matilde qui le voyoit exposé à l'ignominie d'un honteux supplice, fut encore assez genereuse pour demander sa grace au Roy, luy disant qu'il seroit assez puny d'avoir veu ses trahisons découvertes, \& que sa vie, s'il la luy vouloit laisser, luy seroit un supplice continuel qui luy seroit plus sensible que la mort mesme, \& qui ne feroit pas tant de honte à ceux de son sang; enfin à la Priere de cette humaine \& belle Princesse, il fut confiné pour le reste de sa vie dans une Tour du Chasteau, \& on se contenta de faire un exemple de Tebalde par une mort que ses forfaits avoient tres-justement meritée.» (1. 1200-1207).

Boisrobert nous offre ainsi une traditionnelle fin de tragi-comédie, avec un double mariage par le typique procédé de reconnaissances (découverte des fausses lettres d'accusation), mais on voit bien qu'il est obsédé par les gestes exemplaires de magnanimité et d'altruisme de la part de l'héroïne (à l'instar de Théodore), où la bonté et la clémence de la victime éclatent au grand jour. Il nous laisse une image admirable de la femme: bonne, belle, vertueuse et généreuse, mais la nouvelle ne laisse aucune morale par rapport à Fédéric qui à la fin n'est ni puni, ni converti, ni reconnaissant. Rien n'est dit sur son sort, tandis que Roger est pardonné de tous ses crimes. Contrairement à Scarron qui juge en mauvaise part le comportement de ces derniers, chez Boisrobert on est dans la neutralité la plus simple. Concernant le roi, il n'éprouve aucun remord sur son injustice et on ne l'entend jamais s'excuser sur son aveuglément et son extrême crédulité aux propos flatteurs et aux faux rapports de sédition qu'on lui faisait.

\section{La Vie n'est qu'un songe}

C'est un fait avéré et manifeste que cette nouvelle, la quatrième et dernière du recueil de Boisrobert, tirée directement de La vida es sueño de Calderón de la Barca, est aussi la première adaptation de cette œuvre capitale de la littérature espagnole et des plus célèbres de la littérature baroque. Il est intéressant de constater qu'au lieu de faire une traduction littérale du titre de la comedia, Boisrobert en a fait une translation négative à caractère restrictif avec le système corrélatif «ne...que». On verra plus loin qu'on ne doit pas être surpris d'une telle perspective, qui s'explique en bonne partie par la manière dont l'auteur fait son adaptation et l'intérêt tout particulier qu'il porte à présenter un homme dans un état déliquescent, en déchéance, sans illusions et résigné. À croire que l'histoire personnelle de l'écrivain pourrait avoir sa dose de participation dans ce jeu d'adaptations où seraient révélées ses propres déceptions et ses préoccupation datant depuis des années, comme l'injustice des puissants vis-à-vis de leurs favoris, la calomnie et le pardon tardif, et surtout le desengaño ou l'état de désabusement dont on peut être victime par un tour inattendu du destin lorsqu'on s'y attend le moins. Ce qui est certain c'est que Boisrobert reste sensible à cette thématique baroque de retournement de fortune malgré le fait qu'elle commence à passer de mode dans les années 1660. La matière de cette comedia de Calderón contient tous ces ingrédients et s'y prête favorablement, l'adaptation opérée par notre auteur pouvant être alors jugée en ce sens-là tendancieuse, d'autant qu'il revient constamment sur cette problématique qui le hante de manière presque obsessionnelle.

Par ailleurs, la critique est aussi harmonisée sur cet aspect concernant la source, mais le motif central de l'homme qui crois rêver 
alors qu'il agit dans le monde réel n'est pas un sujet exclusif et nouveau au XVIIe siècle, en Espagne notamment pendant sa période de gloire culturelle, et en France aussi pendant sa période dite «baroque», dans la première moitié du siècle. Georges Forestier rappelle dans l'introduction à son édition critique des Songes des hommes esveillez que: "si l'on a à l'esprit que Brosse connaissait fort bien l'œuvre de Calderón, et notamment La Vie est un songe (sic), on ne manquera pas de reconnaître dans le réveil ébloui de Du Pont un travestissement burlesque de la situation dans laquelle Calderón a placé Sigismond. Du Pont, c'est, si l'on veut, Sigismond contaminé par Sancho. Une chose est sûre, en tout cas: Brosse n'a pas inventé le thème de l'homme que l'on tire du néant pour en faire un puissant seigneur, thème qui remonte très haut dans la tradition et qui n'a pas été l'apanage de la seule littérature occidentale ${ }^{48}$ ». Cette œuvre date de 1646, mais ce que dit G. Forestier prouve que Boisrobert n'a pas eu besoin d'y avoir recours par le seul fait qu'elle précède sa propre adaptation de la comedia de Calderón.

Losada Goya fait encore état de la source de notre nouvelle sur laquelle il n'y a pas de zone d'ombre. Mais bien avant lui, G. Hainsworth avait déjà remarqué que la nouvelle de Boisrobert ressemble en tous points à $L a$ vida es sueño, et dans une note il ajoute que: «Boisrobert garde les noms propres employés par Calderón, à l'exception d'Astolfo et d'Estrella, qui deviennent Fédéric et Sophonie (sic); il supprime les rôles de Clarín et de Rosaura ${ }^{49}$ ». À ce stade de ses recherches, le critique ne va pas plus loin, mais dans un article postérieur ${ }^{50}$, il nous renvoie à une étude importante élaborée par Marie Malkiewicz datant de quelques années plus tôt ${ }^{51}$ (nous y reviendrons). Cioranescu s'en fait écho en mettant l'accent sur le fait qu'il s'agit de la première «hypostase française» de la comedia ${ }^{52}$, bien qu'il soit plus sévère et péremptoire dans ces appréciations sur cette nouvelle: "Le travail de rectification consiste à donner à l'intrigue de la comédie les structures propres de la narration, tout en opérant une sélection, très probablement inconsciente, des éléments à conserver. Son choix ne va ni dans le sens des intentions des auteurs espagnols, ni dans celui des tendances du genre, il n'obéit qu'au goût souverain de l'imitateur. C'est le cas par exemple de La vida es sueño, qui n'a que le mérite d'être le premier témoignage français d'un intérêt pour cette œuvre de Calderón. Pour le reste, les questions que celui-ci formule ou suggère, la poésie inquiète qui chante la fragilité du destin, la recherche d'une vérité qui, quoi qu'on fasse, reste une fausse apparence, tout a disparu: Boisrobert n'a aimé que le spectacle de la lutte courtoise entre les deux neveux du roi ${ }^{53}$ »). Exagérés, ces commentaires sont à tous égards réducteurs et il semble que le critique soit passé à côté de la nouvelle, car s'il y a un point sur lequel Boisrobert insiste c'est sur celui des apparences trompeuses, ici et dans quelques-unes de ses pièces de théâtre. C'est d'ailleurs un aspect qui appelle à une étude approfondie.

Deux courtes études supplémentaires font le point sur la question: celle de Monica Pavesio, sur l'héritage de cette comedia en Europe au XVII ${ }^{\mathrm{e}}$ et $\mathrm{XVIII}^{\mathrm{e}}$ siècles ${ }^{54}$, et celle de Daniella Dalla Valle, qui porte sur la toute première imitation de cette œuvre espagnole en France, à savoir la tragi-comédie Les Rivaux Amis (1639) - ne serait-ce qu'en partie ${ }^{55}$ - (et non la nouvelle de Boisrobert, contrairement à ce que l'on pourrait penser), écrite aussi par notre abbé. Par rapport à la pièce de théâtre, on regrette que Mme Dalla Valle reste assez sommaire dans l'analyse de certains aspects essentiels, mais on retiendra un élément intéressant et curieux qui est que Boisrobert connaissait déjà, depuis au moins 1639, cette comedia de Calderón. Mme Dalla Valle montre bien que dans Les Rivaux Amis, notre abbé exploite quelques motifs qu'il développe dans sa tragi-comédie, ce qui vient désamorcer l'injuste accusation selon laquelle il aurait «pillé» les écrits de son frère défunt ${ }^{56}$. Concernant l'étude de Mme Pavesio, la question n'est pas traitée en profondeur non plus, mais elle explique principalement que le thème philosophique et tragique de La vida es sueño nécessitera de la part de Boisrobert d'un traitement tout particulier pour être reçu en France où, à ce stade du siècle, l'on n'est plus trop à même d'apprécier et de comprendre la portée 
de l'œuvre, trop «baroque» sans doute aux yeux des contemporains, à un moment où le fameux «classicisme» est déjà pratiquement intronisé -du moins le prétend-on-, sous-estimant ainsi l'influence qu'exerce encore l'esthétique baroque pendant tout le deuxième tiers du siècle en France. Mme Pavesio rappelle enfin que: «Boisrobert simplifie l'intrigue de la comedia en éliminant l'épisode secondaire historique et géographique, étudie plus profondément le caractère de Sigismond et élimine la trop lourde symbolique baroque ${ }^{57}$ ».

Rappelons que La vida es sueño est le premier poème dramatique inséré dans la Primera parte de la comedias de Don Pedro Calderón de la Barca, publiée en 1636. Son sujet: un prince polonais emprisonné en sauvage depuis son enfance dans les cavernes du château du roi, son père, est assoupi par un breuvage mystérieux et se réveille un jour dans les chambres d'apparat avec toute la magnificence de son rang royal, mais il sera bientôt ramené dans ces cavernes à cause de son comportement brutal, voire sanguinaire, lequel confirmait les prémonitions qui au départ conduisirent son père à le tenir enfermé depuis sa naissance.

Dans La vida es sueño, où sont développés les motifs fondamentaux de la tension binaire entre vie et songe, réalité et illusion, ainsi que le motif de la fatalité de la prophétie et de l'impossibilité de s'y soustraire, Calderón a joué avec la duplicité d'intrigues que Boisrobert essaie de concentrer. Il garde les noms propres employés par Calderón - à l'exception d'Astolfo et d'Estrella, qui deviennent respectivement Fédéric et Sophonie - et supprime les rôles de Clarín et de Rosaura, le but étant de garder une plus grande unité d'action. Celle-ci se concentre en Pologne, à la cour et dans les cavernes du château où Segismundo est prisonnier. Quant à la princesse Sophonie, elle reste «la grande trouvaille» de Boisrobert, pour emprunter les termes de Marie Malkiewicz $^{58}$. Chez Calderón, l'intrigue se trouvait compliquée par la présence de deux femmes: Rosaura, belle Moscovite délaissée par l'infidèle Astolfo (le Fédéric de Boisrobert), qui est venue en Pologne à la recherche de celui-ci; et l'infante Estrella, nièce du roi, prétendante au trône de Pologne, courtisée par Astolfo, mais finalement épousée par Segismundo. Ces deux femmes ont troublé l'esprit de celui-ci au point qu'il hésite toujours entre les deux. Rosaura, déguisée en homme, est la première femme qu'il n'ait jamais vue de sa vie. C'est elle qui éveille en lui un sentiment amoureux jusqu'alors inconnu. Mais lorsqu'il est à la cour, il fait la connaissance de l'infante, qui reste toujours très discrète et cache ses sentiments. Puis, Rosaura, qui a repris ses habits de femme, est devenue la confidente de la princesse, et va réveiller la passion du prince. Ce n'est qu'à regret qu'il la cédera à Astolfo et qu'il se contraint à épouser, comme il se doit, la princesse.

Boisrobert, lui, n'a pas tout à fait construit son histoire de la même manière. Il élimine l'intrigue «secondaire» de La vida es sueño, créant un seul duo amoureux entre Sophonie et Sigismond. Certes, le prince Astolphe est toujours présent dans sa nouvelle, mais il a un rôle de traître et il meurt pour un crime de lèsemajesté, ne laissant ainsi aucun doute sur le fait que son personnage est épisodique. Des deux femmes, Boisrobert fera un seul personnage et lui donnera les traits des deux héroïnes de Calderón, concentrant sur elle tout l'intérêt dramatique. Sophonie sera la première à rendre visite au prince lors de son réveil dans le château royal, et dès le premier moment, les yeux et les cœurs des deux amants se rencontreront pour se comprendre. En ce sens, Boisrobert nous dépeint une histoire d'amour conventionnelle plus qu'une histoire philosophique conforme aux goûts de l'esthétique galante.

Ainsi, selon la formule brillante de Marie Malkiewicz, qui a le mérite d'avoir élaboré l'étude la plus précise et spécifique sur cette adaptation, Boisrobert devait être guidé par l'axiome amo ergo sum, loin de l'idée première de Calderón de nous montrer une histoire philosophique (psychologique, si l'on veut ${ }^{59}$ ), même si les rapports entre le côté romanesque et le côté philosophique sont facilement repérables dans l'adaptation de notre auteur ${ }^{60}$. Il est certain que Boisrobert simplifie l'intrigue, qui est double dans la pièce de Calderón, mais le souci d'unité d'action justifie la concentration opérée par notre 
auteur. Ajoutons que le fait d'avoir consacré sa nouvelle exclusivement à l'histoire de Sigismond, en excluant celle de Rosaura, lui permet aussi d'exploiter l'un des lieux communs qu'il a particulièrement aimé travailler: la difficulté de l'homme à comprendre que la vie est faite d'illusions et de fausses apparences, et que les grandeurs et les vanités humaines sont passagères et peuvent conduire à de graves déceptions et amertumes. C'est ce dont nous avertissait déjà Monica Pavesio ${ }^{61}$, le mérite de Boisrobert étant donc considérable, puisqu'il est le premier à travailler en France l'une des œuvres les plus importantes du répertoire dramatique espagnol. Mme Malkiewics ajoute aussi que: «Précieuse, conventionnelle sans doute, cette histoire d'amour garde un je ne sais quoi d'émouvant et de sincère dans sa simplicité et dans sa logique naïve; aussi Boisrobert, tout bavard et ennuyeux qu'il soit dans sa prose, trouve-t-il quelques notes émouvantes pour rendre les pointes espagnoles et les sentiments des deux amants ${ }^{62}$ 》. Abstraction faite de sa remarque sur le style du nouvelliste, cette critique montre aussi que Boisrobert s'incline davantage, comme il est logique, à développer l'intrigue amoureuse que la réflexion philosophique sur la dualité songe-réalité et s'interroge même si l'attitude de notre abbé n'est pas celle du philosophe matérialiste, avec une pointe d'ironie ${ }^{63}$. S'agissant du personnage de Rosaura, cette idée vient être confirmée du moment où l'auteur soutient l'intrigue amoureuse par une logique plus convaincante que celle de Calderón et par un souci de psychologie assez réussi, susceptible d'être compris et bien reçu de son public.

Concernant la couleur locale polonaise, on ne peut pas reprocher à Boisrobert ce que l'auteur espagnol n'a pas eu la moindre cure de préciser ni de rendre fidèle à la réalité non plus, car le caractère parfaitement flou de la Pologne évoquée se trouve aussi présent chez Boisrobert que chez Calderón. En revanche, il est vrai que par souci de vraisemblance, notre auteur prend beaucoup de temps à nous expliquer le système monarchique de ce lointain pays ainsi que son histoire, qui ayant l'air d'être précise n'est pas moins fausse à plusieurs égards. Nombre de digressions sur ces aspects permettent d'agrémenter son récit, sachant qu'il opère très consciemment des changements ou fait des ajouts qui cherchent à créer une certaine vraisemblance.

Signalons enfin que même dans ce passage du mode dramatique au mode narratif, Boisrobert maîtrise ses sources et fait d'elles ce qu'il veut. Il y a chez lui un vrai travail sur la matière, qu'il réoriente et «acclimate» au goût du public auquel il s'adresse, tout en produisant des nouvelles à la française. Comme le dit A. Cioranescu:

Le travail de rectification consiste à donner à l'intrigue de la comédie les structures propres de la narration, tout en opérant une sélection, très probablement inconsciente, des éléments à conserver. Son choix ne va ni dans le sens des intentions des auteurs espagnols, ni dans celui des tendances du genre, il n'obéit qu'au goût souverain de l'imitateur ${ }^{64}$.

Que l'opération lui paraisse inconsciente, il y a à redire d'autant qu'il est totalement paradoxal que Cioranescu parle de choix. Ce serait aussi mettre hors contexte le travail de l'auteur et l'esthétique galante qu'il élabore et dans laquelle il s'inscrit à la fois. Certes ses adaptations en prose jouissent de beaucoup plus de liberté que son théâtre, elles sont moins réglées, mais il fait un travail tout à fait conscient des matériaux, tout en modulant ses choix et en montrant son talent d'écrivain. Cela ressort encore davantage au vu du corpus dramatique.

\section{Considérations esthétiques concernant les Nouvelles de Boisrobert.}

Les Nouvelles hérö̈ques et amoureuses ont été fort probablement composées juste avant la création de la dernière tragi-comédie de Boisrobert, la Théodore, pièce qui a pu être adaptée à partir de la seconde nouvelle de l'auteur, L'Inceste supposé. Cette pratique n'est pas rare chez lui (rappelons que sa première tragi-comédie Pyrandre et Lisimène est adaptée de son propre roman Histoire indienne), d'autant 
qu'on peut le déduire grâce aux dates «d'achevé d'imprimer», les nouvelles ayant paru le 12 mai 1657 et la pièce de théâtre le 15 novembre de la même année. Cela n'empêche que l'abbé ait pu travailler simultanément à l'écriture des deux œuvres contenu de la proximité temporelle entre la publication des unes et la représentation de l'autre.

À ce stade, on s'aperçoit qu'il n'alterne pratiquement pas le mode dramatique et le mode narratif, sauf pour les deux derniers cas que nous venons d'évoquer avec les réserves qui s'imposent. De même, on a vu que ces nouvelles sont tirées de pièces de théâtre, françaises et espagnoles, et qu'en ce sens on pourrait les appeler des «nouvelles tragi-comiques», à l'instar du recueil de Paul Scarron, ou encore mieux, «désabusements amoureux», tels les Desengaños amorosos de María de Zayas, car toutes les nouvelles présentent une remarquable unité thématique. En effet, la femme y est victime d'accusations calomnieuses, de persécutions, de viols et d'injustices de la part des hommes; aussi $\mathrm{y}$ occupe-t-elle une place essentielle, le thème baroque du desengaño se présentant donc comme corollaire de ces fléaux et traduisant la situation typique du désabusé. C'est l'aspect qui rend le mieux compte de toutes ces œuvres. Boisrobert s'en fait l'écho dans trois récits à l'exemple de la femme écrivain ibérique qui rompt avec les conventions de la nouvelle espagnole de son temps en ce sens qu'elle refuse systématiquement le dénouement heureux en mariage. Chez Zayas, la protagoniste Lisis, désabusée par le comportement brutal et prédateur des hommes, renonce à l'idée d'épouser don Diego et préfère rentrer dans un couvent. Dans ses Desengaños, l'écrivaine s'érige en quelque sorte en l'apologiste de la bonne réputation des femmes et de leur honneur, et tient absolument à les mettre en garde contre les tromperies et la cruauté de la gent masculine:

Y vosotras, hermosas damas, de toda suerte de calidad y estado, ¿qué más desengaños aguardáis que el desdoro de vuestra fama en boca de los hombres? ¿Cuándo os desengañaréis de que no procura más de derribaros y destruiros, y luego decir aún más de lo que con vosotras les sucede?
¿Es posible que, con tantas cosas como habéis visto y oído, no reconoceréis que en los hombres no dura más la voluntad que mientras dura el apetito, y en acabándose, se acabó? [...] ¿Pensáis ser más dichosas que las referidas en estos desengaños? Ése es vuestro mayor engaño; porque cada día, como el mundo se va acercando al fin, va todo de mal en peor. ¿Porqué queréis, por veleta tan mudable como la voluntad de un hombre, aventurar la opinión y la vida en las crueles manos de los hombres ${ }^{65}$ ?

Ce discours aurait pu sembler peut-être trop brutal ou trop réfractaire pour que Boisrobert se l'approprie et s'en fasse écho en France à l'époque. Il se cantonne donc à la fiction, et en ce sens reste assez conventionnel, n'osant pas trop enfreindre les normes sociales en vigueur. Mais il est certain qu'un message allant dans la même perspective, beaucoup moins agressif et direct certes, est transmis à ses lecteurs, et principalement à ses lectrices. Chez Boisrobert, la reine de Maroc et la reine de Hongrie, après les malheurs essuyés, se surpassent elles-mêmes et optent aussi pour une «sainte retraite» à l'abri des contingences de ce monde et de l'injustice aveugle des hommes. Dans Plus d'effets, la princesse de Salerne n'en fera pas autant (c'est une autre solution au même problème) mais elle n'arrive pas moins aux mêmes conclusions et n'est pas moins lucide sur sa fragile situation de femme. Dans La Vie n'est qu'un songe, il s'agit encore d'un desengaño, mais cette fois-ci, il est subi par un homme, le prince Sigismond, qui en fait n'est que le porte-parole de la «pensée» de Boisrobert sur l'amère expérience d'un revers de fortune, thème baroque par excellence que notre auteur se plaît à exploiter dans cette dernière nouvelle, même à l'aube de l'intronisation imminente de l'esthétique classique en France à partir de 1660.

Par ailleurs, on peut dire que dans tous ces récits il est question d'intrigues amoureuses tissées sur un fond politique évanescent qui leur sert de prétexte; elles concernent exclusivement des personnages de très haut rang et ne laissent aucune place à l'élément comique. Elles se déroulent aussi toutes dans un espace géographique exotique autant qu'irréel (même si dans Plus d'effets l'Italie nous est un peu plus familière) et dans une indéfinition temporelle quasi-totale. En revanche, le dénouement frôle 
souvent l'invraisemblance car, contre toute attente, Boisrobert cherche à tout prix à proposer la fin tragi-comique par excellence, qui est celle de l'heureux mariage final, ou comme on l'a dit, la victime des injustices devient presque une image vivante de la Vierge, toute faite de pardon et de rédemption, telle les «femmes fortes» que dépeint le père Le Moyne dans sa célèbre Galerie des femmes fortes. On remarquera cependant que Sigismond fait preuve d'une clémence exemplaire à l'égard des traîtres aussi.

Enfin, contenu du temps relativement court durant lequel l'ensemble de l'œuvre à l'espagnole de Boisrobert a vu le jour, l'on peut dire qu'elle se caractérise par une grande cohérence structurelle et thématique, et que de ce fait elle comporte une grande unité de ton. Elle tourne autour des thèmes de l'amour, de la jalousie, de l'inconstance, de la générosité, du déguisement, de l'engaño (la tromperie), du desengaño (le désabusement) et des apparences trompeuses, que ce soit dans la comédie, la tragicomédie ou les nouvelles. C'est aussi une œuvre qui en général suit d'assez près ses modèles hispaniques tout en opérant des phénomènes d'amplification et de réduction selon l'intérêt que l'auteur porte aux éléments constitutifs de ses sources, le message qu'il veut transmettre à un public susceptible de l'entendre et les exigences normatives du théâtre et de la prose français. Les techniques d'adaptation sont en ce sens récurrentes.

Cela n'empêche pas pour autant que Boisrobert s'adapte lui-même aux goûts d'une certaine tranche du public en ce qu'il n'élimine pas tout à fait le romanesque des œuvres espagnoles et que l'idée de vraisemblance reste assez relative. René Godenne, qui s'est entièrement consacré à l'étude de la nouvelle au XVII ${ }^{\mathrm{e}}$ siècle, montre bien que l'aspect psychologique est fortement développé chez les auteurs en général mais qu'il n'est pas moins vrai que les rebondissements et les accidents de toute nature, à l'instar de la tragicomédie du premier tiers du siècle, sont à l'ordre jour avec autant de vigueur. C'est ce qu'il explique en ces termes: « la nouvelle des années 1600-1750 n'est pas un récit où prédomine une intension d'ordre psychologique, mais une narration qui se définit par son caractère romanesque. Tout se passe comme si les nouvellistes s'appliquaient à retrouver et exploiter le fond d'aventures extraordinaires, voire héroïques des œuvres longues de la première moitié du XVIIe siècle. Les auteurs sont incapables, semble-t-il, de concevoir le développement d'une intrigue sans ses incidents 'classiques' que sont les rapts d'héroïnes, les évasions des héros du sérail, les naufrages de vaisseaux dus à des tempêtes aussi nombreuses que subites, les attaques de corsaires, de pirates, les substitutions de personnes, les reconnaissances inattendues, les déguisements, etc. À cet égard, les entrées en matière nous installent dans un monde où le romanesque est roi $^{66}[\ldots] \gg$.

Comme dans le cas de Boisrobert, les nouvellistes placent le sujet des intrigues dans les milieux de la haute aristocratie, comme on faisait dans les romans dits «baroques». Cet environnement permet de ressortir l'esprit galant plutôt que le désir de montrer un ordre hiérarchique sociale, ce qui dans le contexte dans lequel notre auteur fait paraître ses nouvelles est tout à fait logique, car ce sont encore des valeurs prisées par la société galante qu'il fréquente et à laquelle il adresse son œuvre. Ainsi, les jeux de société sont également présents dans les récits, dans lesquels participent les personnages pour montrer leur attachement chevaleresque pour l'héroöne et l'être aimé, d'où les divertissements comme les jeux de bague que l'on voit dans Plus d'effets que des paroles (où don César étale ses remarquables dons pour la course à cheval), et aussi toute une série de marques de galanterie comme les lettres et les billets que l'on lit ici et là, ou encore des bribes de poèmes (sonnets, madrigaux, etc.), sans parler des jeux de déguisement et de changement d'identités, où le valet se fait passer pour son seigneur de manière à essayer de détourner les intentions d'une reine qu'on n'aime pas (dans L'Heureux Désespoir) ou encore, quand un seigneur se déguise en simple jardinier pour approcher la belle dont l'accès lui est interdit (idem). Autant de transpositions qui font que ce jeu soit considéré comme galant. Godenne croit d'ailleurs que l'introduction de la galanterie dans la nouvelle conduit à ce que 
ce genre baigne encore dans l'invraisemblance et reste dans l'esprit dit «précieux » du premier tiers du siècle ${ }^{67}$. C'est pourquoi, dépourvu à ce stade de l'évolution du genre, de son caractère de récit oral comme aux $\mathrm{XV}^{\mathrm{e}}$ et $\mathrm{XVI}^{\mathrm{e}}$ siècles, la nouvelle est désormais parée des ornements de la galanterie et se dirige vers une autre conception esthétique.

Et qui dit adaptation dit se placer par rapport à l'autre. Boisrobert signale dans la dédicace de ses Nouvelles hérö̈ques à Nicolas Foucquet, son dernier puissant protecteur, que:

Les sujets que j'ai tirés tout nus et tout simples de l'Espagnol, et que j'ai rectifiés selon nos manières, en sont tout particuliers et tout beaux, les incidents en sont tout merveilleux et tout surprenants, le style en est tout pur et net, et enfin ce petit ouvrage peut passer parmi les plus délicats de la cour pour galant et pour agréable.

Du côté de la source, rien à dire: Boisrobert reconnaît que l'invention est espagnole. Cependant, l'aspect formel pose problème: il faut le rectifier, d'après lui. Mais ce souci de «rectification» ne lui est pas exclusif à Boisrobert: l'époque le veut, et le croit, et les doctes français l'exigent. On va constater que les hommes de lettres du temps, qui considèrent la littérature espagnole comme déréglée et obscure, vont essayer de soumettre leurs œuvres aux exigences des règles des unités, de bienséance et de vraisemblance, ce qui est très discutable comme on l'a vu précédemment. Boisrobert ne fait pas exception, notamment lorsqu'il indique plus tôt dans la préface La Folle Gageure que:

Quoique la gloire de l'invention soit due au fameux Auteur Espagnol d'où j'ai tiré le sujet de cette Comédie, je prétends toutefois, si elle mérite quelque louange, que j'y dois prendre quelque part, puisque non seulement j'en ai retranché toutes les choses importunes et superflues, qui faisaient peine à l'esprit, mais que je pense encore en avoir rectifié plusieurs autres qui faisaient autant de peine au jugement. S'il te plaît, Lecteur, te donner la peine de lire cette Comédie dans l'Espagnol, sous le titre qui lui est donné du Plus grand impossible ${ }^{68}$, tu m'avoueras que je n'ai pas fait un petit effort de l'avoir en quinze jours si proprement habillée à la Française, et le Fripier ne te paraîtra peut-être pas moins adroit que le Tailleur.
Tu en trouveras une autre chez le même Libraire nouvellement imprimée, sous le titre des Trois Orontes, qui est toute de mon esprit et de ma façon, et par elle tu jugeras, que si nous nous donnions quelquefois la peine d'inventer, les Espagnols ne seraient pas les seuls maîtres des belles inventions.

Je t'en promets une dans fort peu de temps que j'ai tirée du même Auteur Espagnol, sous le titre de La Vérité menteuse ${ }^{69}$. Je me suis plusieurs fois étonné en la lisant, comment les illustres Corneille [...] n'ont point découvert celui-ci si plein de richesse et d'invention. Je puis dire avec vérité que le grand Lope de Vega s'y est surmonté lui-même. Je ne vis jamais rien de si beau ni de si brillant, mais j'ose croire sans beaucoup de présomption que je l'ai rendu juste et poli, de brut et de déréglé qu'il était, et que si nos Muses ne sont pas aussi inventives que les Italiennes et les Espagnoles, elles sont au moins plus pures et plus réglées ${ }^{70}$.

Les remarques faites ici sont révélatrices de cet état d'esprit, car la vision que l'on a de la littérature espagnole paraît transparente: elle est brute et irrégulière. On retiendra quand même que Boisrobert, comme beaucoup de ses contemporains, reconnaît sans ambages le génie inventif des auteurs hispaniques, et c'est ce qui les conduit à les chercher. Mais il est manifeste que la littérature espagnole nécessite pour eux d'importants remaniements avant d'être produite et transmise à la culture française.

On notera que les nouvelles de Boisrobert n'ont pas un encadrement dans lequel elles sont récitées contrairement à la tradition instaurée par Boccace et suivie en France même avec Sorel, où sous prétexte d'être surpris par une tempête, une escale dans un château, ou autres déconvenues, les personnages sont obligés de raconter des histoire pour tuer le temps et passer un bon moment ensemble. On a déjà vu que María de Zayas et Alonso del Castillo Solórzano, où Boisrobert vient en partie puiser son œuvre, rejoignent cette pratique, eux qui sont beaucoup moins préoccupés que leur maître Cervantès de maintenir la vraisemblance dans leurs récits, comme le rappelle G. Hainsworth dans sa remarquable étude sur les Nouvelles exemplaires de Cervantès en France au XVII ${ }^{\mathrm{e}}$ siècle $^{71}$.

Ces œuvres se caractérisent par l'exotisme spatio-temporel. On est d'abord à la brillante cour 
de Grenade chez Boabdil Nasride, pour passer ensuite dans les cours du Saint-Empire (Hongrie notamment), et plus au nord ensuite à Cracovie dans les domaines du roi Basile (nom pourtant plus adapté aux monarques Moscovites), pour descendre enfin en Italie, sur les rivages de la côte amalfitaine où la princesse Matilde est malmenée par son cousin qui désire lui ravir sa principauté de Tarente.

Pourtant, si le vraisemblable devient un critère fondamental dans la mentalité de l'époque, les auteurs français ne se privent pas d'avoir recours à des nouvellistes espagnols comme Montalbán, par exemple, qui est parmi tous, des plus invraisemblables qui soient, notamment lorsqu'on sait que ses œuvres peuvent baigner dans le merveilleux le plus pur (voir Sucesos y prodigios de amor, 1624). À ce sujet, G. Hainsworth signale que «la popularité de ces nouvelles est attestée d'ailleurs par diverses imitations, et plus tard, par de nouvelles traductions, d'après lesquelles il n'est pas difficile de deviner que Montalvan (sic) a plu grâce précisément au romanesque que nous lui avons reproché72 $\gg$.

Dans les processus d'adaptation français, la couleur locale espagnole s'est estompée pour céder la place à des lieux beaucoup plus exotiques dans le goût romanesque le plus pur. Mais c'est en 1651, avec la publication de la première partie du Roman comique de Scarron que commence en effet une nouvelle étape dans la traduction et l'adaptation de la nouvelle espagnole en France. Cet auteur ne tarde pas à être secondé par D'Ouville et ensuite par Boisrobert, donnant, de 1651 à 1657 , une série de nouvelles espagnoles empruntées surtout à deux auteurs, Castillo Solórzano et María de Zayas, comme on l'a assez fait voir mais qui étaient pratiquement inconnus des lecteurs français.

On peut dire que c'est à Scarron que l'on doit la théorisation de cette nouvelle vague d'adaptations en mode narratif, à partir d'une série de remarques que l'on entend dire à un des personnages de son Roman comique, «un jeune conseiller du parlement de Rennes», des remarques qui montrent bien que dans le choix de ses sources, il s'appose aux travers du roman héroïque trop outrés de ses prédécesseurs.
D'ailleurs, Scarron se caractérise par une prise de distance remarquable, donnant un tour très particulier aux œuvres qu'il adapte, mais il faut dire qu'au fond il ne s'accommode pas moins de la littérature romanesque espagnole qui lui plaît tant. Ce qui est certain c'est qu'il cherche à abréger, ce en quoi il est suivi par ses confrères, et notamment par Boisrobert, et s'attaque aux longues descriptions des romans à la mode, de manière à créer une sorte de complicité avec son lecteur. C'est ainsi qu'on peut entendre des propos de la sorte dans son Roman comique qui vont dans le même sens:

« les auteurs français devraient essayer de supplanter les romans héroïques en écrivant, à l'exemple des Espagnols, des nouvelles romanesques»,

il existe une bonne et une mauvaise façon d'écrire de romans et des nouvelles,

il y a une prééminence des Espagnols dans le domaine de la nouvelle,

leurs récits ont des sujets bien plus vraisemblables que ceux qu'on trouve dans les romans héroïques français, les Novelas exemplares de Cervantès étant le modèle par excellence,

on préférera des histoires comme l'Histoire de l'amante invisible, À trompeur, trompeur et demi, Le Juge de sa propre cause et Les Deux Frères ennemis que l'on trouve insérées dans Le Roman comique, autant de «traductions» ou d'adaptations (pour être plus justes) qui laissent supposer qu'elles seraient les modèles de nouvelles «bien faites» par les Espagnols et qui se prêtent à une adaptation aisée en France.

la nouvelle idéale est caractérisée par une intrigue bien nouée, et par un romanesque dont les données sont concevables, et qui promet mieux que le roman héroïque ou les récits extravagants à la manière de Montalbán.

De Zayas et Solórzano, où Scarron puise la plupart de ses récits, loin de prendre ce 
qu'ils ont de plus romanesque, il a soin de ne traduire que des nouvelles d'une vraisemblance relative ${ }^{73}$.

Rappelons que les œuvres adaptées de Scarron, dont le rire est d'une force indéniable même encore aujourd'hui, portent la marque indélébile du burlesque; ses qualités sont le réalisme et l'ironie, et il doit beaucoup à Sorel mais surtout à Cervantès, qui a modelé son tempérament. Hainsworth considère cependant que Cervantès reste plus subtile que Scarron tandis que Sorel tombe souvent dans le pédantesque ${ }^{74}$.

On constatera par ailleurs que ce qui intéresse dans les nouvelles de Solórzano c'est le cadre à l'italienne et l'emploi d'intrigues ingénieuses et compliquées, motivées par les sentiments d'honneur ou d'amour, tandis que María de Zayas élabore des nouvelles romanesques à souhait, où il est question de travestissements, de magie et de surnaturel. Chez elle, tout baigne dans le merveilleux le plus pur. Ses nouvelles sont destinées à « montrer la méchanceté du sexe masculin, et leurs données, assassinats, viols, vices contre nature, envoûtements, ont en général un caractère tragique ou macabre », comme l'a bien remarqué G. Hainsworth, qui ajoute que: «il est question, dans la plupart d'entre elles, des crimes des parents effroyablement jaloux, qui s'acharnent à punir, non seulement par la mort, mais par des tortures atroces, des femmes innocentes, ou qui n'ont péché qu'à leur corps défendant ${ }^{75}$ \%. $\mathrm{Ce}$ sont des thèmes auxquels Boisrobert est extrêmement sensible et qui lui sont servis sur un plateau d'argent pour les reproduire en mettant l'accent sur ces aspects, sachant qu'en France il existe un public susceptible d'entendre de bon cœur ces discours. Scarron, qui puise chez ces Espagnols aussi, procède pourtant différemment, en conférant des limites au romanesque outrée que l'on y trouve, et selon le goût de Cervantès. D'ailleurs, il ne se prive pas de faire ses propres ajouts et commentaires, qui s'éloignent même de l'esprit grave et posé de la source ${ }^{76}$. Mais on remarquera que ces nouvelles ont toute en commun le fait qu'un personnage principal, ayant adopté un déguisement, donne lieu à une série de malentendus qui se multiplient crescendo jusqu'au point où la tension tombe au dénouement grâce au typique procédé de reconnaissances. Ajoutons que dans le cas de Scarron, il opère aussi de nombreuses interpolations de son cru qui vont dans le sens de corriger l'excès de romanesque que l'on trouve dans les sources espagnoles, par un souci de réalisme relatif. Mais contrairement à ce que pense G. Hainsworth, ce ne sont pas des «correctifs», car on aurait mauvaise grâce de considérer que l'œuvre espagnole est mal conçue, puisqu'elle n'existe que par rapport à ses propres données poétiques, esthétiques et rhétoriques. Très différent est le regard que portent sur elles les Français de l'époque, qui ne font qu'interpréter les sources à leur guise, et par rapport à leurs propres besoins et schémas de pensées, dans le but d'assouvir et de s'adapter à leur public et à leurs propres réalités culturelles et sociales.

Les Nouvelles exemplaires et amoureuses de D'Ouville et les Nouvelles héroïques et amoureuses de Boisrobert, que G. Hainsworth estime de moindre intérêt que celles de Scarron, sont postérieures à la première partie du Roman comique et aux Nouvelles tragi-comiques, parues au cours de 1655. L'on sait que D’Ouville reproche à ce dernier les écarts qu'il se donne la liberté de prendre par rapport aux Espagnols, mais en retour il se fait accuser d'être trop servile traducteur. Car en effet, le frère de Boisrobert est beaucoup plus fidèle à la source qu'il s'efforce de respecter sans toutefois y parvenir. Pour sa part, Boisrobert fait un contraste encore plus frappant avec Scarron (D’Ouville étant plutôt au milieu puisque celui-ci reste plus neutre dans ses traductions), car Boisrobert ajoute le caractère d'esthétique «galante» à ses adaptations. Il est en effet l'antithèse de Scarron. Il s'éloigne sciemment de tout ce qui est bas et grotesque, de tout réalisme cru et terre à terre indigne des personnages de haut rang qui peuplent ces nouvelles. Le style galant ${ }^{77}$ y est à l'ordre du jour et même les rares personnages de bas rang que 
l'on y rencontre parlent aussi sur ce ton. La satire et le burlesque seraient des intrus dans l'univers boscorobertin. Il reste ainsi un fidèle partisan du romanesque héroïque à la manière des grands romanciers du premier tiers du siècle.

Nous avons donc chez notre abbé cette combinaison de galanterie qui relève des intrigues amoureuses qu'il développe dans ses nouvelles et d'héroïsme propre aux personnages extraordinaires, capables de surmonter les plus dures épreuves et d'entreprendre les plus éprouvantes aventures. L'abbé revendique ce romanesque, qui n'est pas manifestement mort vers 1660 , mais qui au contraire vient satisfaire le goût d'une bonne partie du lectorat de l'époque. Il accentue même ce côté galant et romanesque par les mésaventures que subissent les héroïnes de ces histoires, ce qui lui permet d'exalter leur image par l'exemple de magnanimité qu'elles donnent à la fin, de détachement, de dépassement de soi, d'altruisme même, et du sens profond de justice, très à l'encontre des réalités de l'époque. Exalter la femme dans cette œuvre laisse manifeste que c'est à celle-ci qu'il s'adresse essentiellement, elles qui font la réputation des hommes dans les ruelles et qui sont capables de donner du prix à l'art littéraire.

Les sujets adaptés par Boisrobert relèvent de ce souci majeur, et c'est ce qu'on remarque aux titres qu'il a choisis pour ses adaptations. L'Heureux Désespoir, qui ressemble au titre de la source L'Heureuse Constance de Rotrou, renvoie au désespoir qu'éprouve notamment le héros, Abindare, d'être confronté à une infinité d'obstacles pour rejoindre la belle Cheriffe, qui semble lui échapper à tout moment, mais qui lui revient à la fin grâce à la constance amoureuse dont il ne se dément jamais, et qui lui permet de l'épouser. De même L'Inceste supposé, qui empruntele titrehomonyme de la pièce dramatique de La Caze, révèle cette préoccupation majeure qui hante Boisrobert par rapport à l'injustice dont sont victimes les humains, et surtout les femmes. Car cet «inceste» qui n'est que supposé vaut des persécutions effroyables à l'héroïne qui ne peut compter après tout que sur elle-même et sur la grâce divine. Pour sa part, le titre de Palabras y plumas (paroles et plumes) de Tirso trouve son écho dans la translation opéré par Boisrobert à travers Plus d'effets que de paroles, que Scarron avait déjà crée pour sa propre adaptation de la comedia de l'Espagnol. Boisrobert se l'approprie en se montrant son franc concurrent pour donner un tour très différent à celui de Scarron à son adaptation. Pourtant, le titre traduit parfaitement le fond de la problématique: un amant extravagant, prétentieux, lâche et jaloux ne fait que promesses et bravades mais n'en vient jamais aux faits pour secourir la princesse en danger qu'il est sensé aimer et épouser. Mais si le titre de Tirso suggère plus les défauts de cet amant antihéros, Scarron et Boisrobert mettent l'accent sur le vrai héros de la nouvelle: le cavalier espagnol qui agit en vrai chevalier médiéval, au service de sa dame, capable de toute entreprise dangereuse pour elle, et d'une constance amoureuse à toute épreuve à l'instar d'Amadis de Gaule. Ce dernier ne tombe pas dans les «paroles et les plumes» comme son rival, mais on le voit produire plus d'actes concrets (effets) que des promesses dites en l'air (paroles). Enfin, La Vie n'est qu'un songe est étonnement une translation restrictive en mode négatif de la source qui emploie un énoncé affirmatif : La vida es sueño. Ce sujet, dont la portée philosophique est essentielle dans la comedia espagnole de Calderón est plus réduit chez Boisrobert pour donner une intrigue amoureuse plus relevée. Mais cela suppose auparavant que les amants subissent des mésaventures qui leur font arriver à la conclusion désabusée que la vie ne se réduit qu'à un songe, et que les gloires dont on peut jouir à un moment donné peuvent se transformer à tout moment en source de souffrance et en de cruelles infortunes, créant ainsi un état de désabusement cuisant chez l'individu. C'est pourquoi, malgré l'invraisemblance qui hante ces œuvres, elles ne sont pas moins recevables, et même avec enthousiasme, car le caractère moralisant en est manifeste, et on est invité à en dégager des leçons de vie.

Tant est vrai que la fortune de la nouvelle en France ne se démentira pas, car on verra paraître toute une série d'adaptations comme celles de Vanel, à partir de María de Zayas, en 1680 et 1683 . Les nouvelles de Scarron seront 
sans cesse réimprimées au XVIII ${ }^{\mathrm{e}}$ siècle, et les apports de D'Ouville et de Boisrobert ne viennent qu'à enrichir et consolider la tendance ${ }^{78}$, dont témoignent aussi les nouvelles de Segrais (Nouvelles françoises), L’Amant ressuscité, nouvelle de Me A. Ancelin et Le Rival encore après la mort, anonyme, toutes datant d'avant 1660. C'est cela aussi qui a permis d'éclipser la force qu'avait eu auparavant le roman, obligé d'opérer des modifications fort considérables pour subsister auprès du public. Par la suite, le souci d'un Segrais sera tout de même de dépouiller de plus en plus la nouvelle de son romanesque et de son invraisemblance pour la rendre plus proche de la vie quotidienne, ainsi que l'exprime la princesse Aurélie: «C'est la différence qu'il y a entre le roman et la nouvelle... que la nouvelle doit un peu davantage tenir de l'histoire, et s'attacher plutôt à donner les images des choses comme d'ordinaire nous les voyons arriver, que comme notre imagination se les figure ${ }^{79}$ ». Le travail adaptatif de Boisrobert à travers ses nouvelles, au regard de cette citation, semble ainsi programmatique.

\section{Orientation Bibliographique}

Adam, Antoine. (1997). Histoire de la littérature française au XVII siècle (1948). Paris, Albin Michel, coll. Bibliothèque de l'Évolution de l'Humanité.

Arellano-Ayuso, Ignacio. (2005). Historia del teatro español del siglo XVII (1995). Madrid, Cátedra, coll. Crítica y estudios literarios.

Baudeau de Somaize, Antoine. (1657). Remarques sur la Théodore de l'Autheur de Cassandre. Paris, à ses dépends.

Birkemeier, Sven. (2007). Rotrou adaptateur de la comedia espagnole, thèse de l'université de Münster.

Bourqui, Claude. (2006). La transmission des sujets galants hispaniques à la scène française du XVII ${ }^{\mathrm{e}}$ siècle: hypothèse sur le rôle du Grand Cyrus. Papers on French Seventeenth Century Literature, éd. Rainer Zaiser, Tübingen, Gunter Narr, « Biblio 17 », 33(64), 97-108.

(1994). Une source peu connue de Molière: le théâtre de Le Métel D’Ouville. Visages du théâtre français au XVII siècle. (115-127) Paris, Klincksieck.

(1996). D’Ouville adaptateur du théâtre espagnol: deux sources nouvelles, $X V I I^{e}$ siècle, 191 (avril-juin), 359-363.

Chardon, Henri. (1904). Scarron inconnu. Paris, Champion, 2 vol.

Cioranescu, Alexandre. (1969). Bibliographie de la littérature française du XVII siècle. Paris, CNRS.

(1983). Le masque et le visage. Du baroque espagnol au classicisme français. Genève, Droz.

(1977). Bibliografía francoespañola, Madrid, Real Academia española.

. (1957). El barroco o el descubrimiento del drama. La Laguna, Secretariado de Publicaciones de la Universidad de la Laguna, 1957.

(1954). Estudios de literatura española y comparada, La Laguna de Tenerife, Universidad de La Laguna, Secretariado de publicaciones.

(1966). «La nouvelle française et la Comedia espagnole au XVII siècle», La Nouvelle en France jusqu'au XVIIIe siècle. Maximes et portraits. Poésie et peinture en France au XIXe siècle, Cahiers de l'Association internationale 
des Études françaises (CAIEF), Paris, Les Belles lettres, 18(mars), 79-87.

Cobos-Castro, Esperanza. (1992). Las fuentes españolas de Plus d'effets que de paroles de Boisrobert, Estudios de investigación franco-española, 6, 33-90.

Conesa, Gabriel. (1989). Pierre Corneille et la naissance du genre comique (1629-1636), Paris, Sedes, coll. Critique littéraire.

Couderc, Christophe. (1997). Le système des personnages de la Comedia espagnole (1594-1630). Contribution à l'étude d'une dramaturgie, thèse de doctorat dirigée par Jean Canavaggio, Université de Paris X-Nanterre.

(2006). Galanes y damas en la comedia nueva: una lectura funcionalista del teatro español del Siglo de Oro. MadridFranckfurt am Main, IberoamericanaVuervert.

(1998). La comedia de enredo y su adaptación en Francia en el siglo XVII: transformación de un género. La Comedia de enredo: Actas de las jornadas de teatro clásico, 1997, Almagro 8-10 de julio, éd. Felipe B. Pedraza Jiménez et Rafael González Cañal, Almagro, Universidad de Castilla-La Mancha et Festival de Almagro, coll. Corral de comedias, 269-283.

Coudert, Marie-Thérèse. (2004). Traduire, adapter, représenter la Comedia: pour une contribution à l'étude de la réception $d u$ théâtre espagnol du Siècle d'or en France, thèse de doctorat de l'Université de Paris X-Nanterre, dirigée par Jean Canavaggio.

Crepet, Eugène. (1861). Les poètes français. Paris, Gide Libraire.
Dalla-Valle, Daniela. (1998). La prima imitazione francese della Vida es sueño e la lettura barocca dell'Edipo: Les Rivaux amis di Boisrobert, Rivista di letterature moderne e comparate, Firenze, Pacini, 51 (3 juilletseptembre), 281-291.

Deierkauf-Holsboer, Sophie-Wilma. (1970). Le Théâtre de l'Hôtel de Bourgogne. Le Théâtre de la troupe royale (1635-1680), Paris, Nizet, 2 vol.

Denis-Delanda, Delphine. (1996). Réflexions sur le 'style galant': une théorisation floue, Littératures Classiques. Le Style au XVIIe siècle, 28, 147-158.

Dumas, Catherine. (2004). Du Gracioso au valet comique. Contribution à la comparaison de deux dramaturgies (1610-1660). Paris, Honoré Champion.

Émelina, Jean. (1975). Les valets et les servantes dans le théâtre comique en France de 1610 à 1700. Grenoble, Presses Universitaires de Grenoble.

Fatio-Morel, Alfred. (1923). La Comedia espagnole $d u$ XVII e siècle. Leçon d'ouverture. Paris, Honoré Champion.

Gilot, Michel et Serroy Jean. (1997). La comédie classique en France. Paris, Belin, coll. Lettres Sup.

Godenne, René. (1974). La nouvelle française, Paris, PUF, coll. SUP. Littératures modernes.

Guevara-Quiel, Francisco. (2009). Édition critique du théâtre et des nouvelles " à l'espagnole " de l'abbé Boisrobert, Thèse de doctorat de l'Université de Paris IV-Sorbonne, dir. Georges Forestier, Paris.

(janvier-juin 2011) Les Nouvelles héroïques et amoureuses de l'abbé 
Boisrobert, Revista de Lenguas Modernas, San José, Escuela de Lenguas Modernas, Université de Costa Rica, 14, 55-74.

Guichemerre, Roger. (1981). La Tragi-comédie, Paris, Presses Universitaires de France, coll. Littératures Modernes.

. (1972). La Comédie avant Molière (16401660), Paris, Armand Colin.

(1991). La francisation de la Comedia espagnole chez d'Ouville et Scarron, dans L'âge d'or de l'influence espagnole. La France et l'Espagne à l'époque d'Anne d'Autriche, 1615-1666. Actes du 20 e Colloque du Centre Méridional de Rencontres sur le XVII siècle (CMR 17), Bordeaux, 25-28 janvier 1990, éd. Charles Mazouer, Mont-de-Marsan, Éditions Interuniversitaires, 255-268.

. (1994). Les nouvelles du Roman comique de Scarron et la comédie «à l'espagnole, Visages du théâtre. Mélanges offerts en l'honneur de Roger Guichemerre, dir. Forestier Georges et Haroche-Bouzinac Geneviève, Paris, Klincksieck, coll. Bibliothèque de l'âge classique, 263-274.

(1994). Une source peu connue de Molière: le théâtre de Le Métel d'Ouville, Visages du théâtre. Mélanges offerts en l'honneur de Roger Guichemerre, dir. Forestier Georges et Haroche-Bouzinac Geneviève, Paris, Klincksieck, coll. Bibliothèque de l'âge classique, 57-69.

(1994). De théâtre au roman: Palabras $y$ plumas, de Tirso de Molina, et Plus d'effets que de paroles, une nouvelle tragicomique de Scarron, dans Visages $d u$ théâtre français au XVII siècle, Paris, Klincksieck, 275-279.

Hainsworth, George. (1947). New Details on the Nouvelles of Scarron and Boisrobert,
Bulletin Hispanique, Bordeaux, 49(2), 145-149.

(1971). Les Novelas exemplares de Cervantès en France au XVII siècle. Contribution à l'étude de la Nouvelle en France (1933), New York, Burt York, réimpr. éd. de Paris, chez Champion.

Hildesheimer Françoise. (2000). Du Siècle d'or au Grand Siècle. L'État en France et en Espagne, XVIe-XVIIe siècle, Paris, Flammarion, coll. Campus Université.

Hippeau Célestin. (1858). Les écrivains normands au XVIIe siècle. Caen, Bohour.

Hubert M. J. D. (1966). Les nouvelles françaises de Sorel et de Segrais. La Nouvelle en France jusqu'au XVIII siècle. Maximes et portraits. Poésie et peinture en France au XIX $X^{e}$ siècle, Cahiers de l'Association internationale des Études françaises (CAIEF), Paris, Les Belles lettres, 18(mars), 31-40.

Iline Anastasia. (2004). François Le Métel de Boisrobert (1592-1662). Écrivain et homme de pouvoir, Paris, École Nationale de Chartes, Thèse pour le diplôme d'archiviste paléographe, 3 vol.

King, Katherine. (1975). Boisrobert's Nouvelles héroïques et amoureuses and the Histoire Indienne: his prose adaptations from the spanish, Thèse du Département des Langues étrangères, Université de Louisiana.

Klein, Andras. (1996). Une tragi-comédie française sur un sujet hongrois: Boisrobert : Théodore, reine de Hongrie, Revue d'Études françaises, Budapest, Département d'études françaises et le Centre interuniversitaire d'études françaises de l'Université de Eötvös Loránd de Budapest, 2, 177-209. 
Labitte, Charles. (1857). Études littéraires, avec une notice biographique et bibliographique sur les personnages nés en Normandie et sur ceux qui sont seulement distingués par leurs actions ou par leurs écrits, Rouen, A. Le Brument.

Lancaster, Henry Carrington. (1929-1942). A History of French Dramatic Literature in the Seventeenth Century, Baltimore, The John Hopkins Press, 5 part., 9 vol.

Larroumet, Gustave. (1892). Un historien de la société précieuse au XVIIe siècle : Baudeau de Somaize, Revue des deux mondes, 92, 125-155.

Lopera-Rodríguez, Laura M. (1998). Sobre la configuración del género Novella en la literatura francesa: el caso concreto de El Castigo de la miseria de Zayas y Le Chastiment de l'avarice de Scarron ", Estudios de Investigación franco-española (EIF), Córdoba, Estudios de Investigación franco-española de la Universidad de Córdoba, 15, 51-72.

Losada-Goya, José Manuel. (1999). Bibliographie critique de la littérature espagnole en France au XVIIe siècle, présence et influence, Genève, Droz.

(1994). L'honneur au théâtre. La conception de l'honneur dans le théâtre espagnol et français du XVIIe siècle, Paris, Klincksieck.

Mac-Curdy, Raymond. (1992). Historia y crítica de la literatura española. Siglos de Oro, Barroco, éd. Francisco Rico et Carlos Vaíllo, Barcelona, Crítica.

Malkiewicz, Marie. (1939). Un remaniement français de La Vie est un songe (sic), Revue de Littérature comparée, XIX, 429-444.
Marchal-Weyl, Catherine. (2007). Le Tailleur et le fripier. Transformations des personnages de la comedia sur la scène française (16301660), Genève, Droz.

Martinenche, Ernest. (1990). La Comedia espagnole en France de Hardy à Racine, Paris, Hachette.

Maurel, Serge. (1971). L'univers dramatique de Tirso de Molina, Poitiers, Publications de l’Université de Poitiers.

Mazouer, Charles. (1991). L'âge d'or de l'influence espagnole. La France et l'Espagne à l'époque d'Anne d'Autriche, 1615-1666. Actes du 20e Colloque du Centre Méridional de Rencontres sur le XVIIe siècle (CMR 17), Bordeaux, 25-28 janvier 1990, éd. Charles Mazouer, Montde-Marsan, Éditions Interuniversitaires.

Merino-Garcia, Daniela. (2005). De la comedia à la nouvelle : Tirso de Molina en Francia, Actes de la première rencontre hispanofrançaise de chercheurs, Séville. Document en ligne : http://www.culturadelotro.us.es/ actasehfi/pdf/2merinog.pdf. Consulté le 14.09.2012.

Morillon, Paul. (1888). Scarron et le genre burlesque, Paris, Lecène et Oudin.

, Scarron, (1888). Étude biographique et littéraire, Paris, Lecène et Oudin.

Pageaux, Daniel-Henri (dir.). (Juin 1984). Deux siècles de relations hispano-françaises. De Commynes à Madame d'Aulnoy. Actes du colloque international du CRECIF (Centre de recherches et d'études comparatistes ibéro-francophones de la Sorbonne-Nouvelle), Paris, L'Harmattan, coll. Récifs. 
Pavesio, Monica. (2000). Calderon in Francia: ispanismo e italianismo nel teatro francese del XVII secolo, Torino, Ed. dell'Orso.

(1994). La vida es sueño dans la littérature française au XVIIe et XVIIIe siècles, Estudios de Investigación francoespañola, 10, 85-95.

Picciola, Liliane. (2002). Corneille et la dramaturgie espagnole, Tübingen, Gunter Narr.

Pure, Michel (Abbé de). (1938). La Pretieuse ou Le Mystère des ruelles. Dediee a telle qui n'y pense pas, (Paris, Pierre Lamy, 1656), La Pretieuse ou Les Mysteres de la ruelle. Seconde partie, (Paris, Pierre Lamy, 1656), éd. Émile Magne, Paris, Droz.

. (1939). La Pretieuse ou Le Mystère des ruelles. Troisiesme partie (Paris, Guillaume de Luyne, 1657). Le Roman de la Prétieuse ou Les Mystères de la ruelle. Quatriesme et derniere partie (Paris, Guillaume de Luyne, 1658), éd. Émile Magne, Paris, Droz.

Reynier, Gustave. (1914). Le roman réaliste au XVIIe siècle. Paris, Hachette.

Ruano de La Haza, José María. (2000). La puesta en escena en los teatros comerciales del Siglo de Oro, Madrid, Castilia.

Schaub, Jean-Frédéric. (2003). La France espagnole. Les racines de l'absolutisme français, Paris, Seuil, coll. L’Univers historique.

Suc, Jean-Claude. (1981). Les Premières Comédies françaises imitées de Calderón : début d'une vogue théâtrale lancée par D’Ouville (1639-1651), Université de Lyon.

Urbain, Henri. (1970). La mode espagnole dans la comédie en France de 1645-1656, thèse de l'Université de Berkeley, California.

Viala, Alain. (1985) Naissance de l'écrivain. Sociologie de la littérature à l'âge classique, Paris, Éditions de Minuit.

Vitse, Marc. (1990). Éléments pour une théorie $d u$ théâtre espagnol du XVIIe siècle, Toulouse, Presses Universitaires du Mirail.

(1995). La poética de la Comedia: estado de la cuestión o de la poética a las poéticas de la Comedia. La Comedia : Seminario hispano-francés organizado por la Casa de Velázquez diciembre 1991-junio 1992, éd. Jean Canavaggio, Madrid, Casa de Velázquez, 273-289.

Warshaw, J. (1913). The case of Somaize, Modern Language Notes, 28(2), 33-38.

. (1914). The identity of Somaize I, MLN, 29(2), 33-36.

(1914). The identity of Somaize II, $M L N$, 29(3), 77-82. 
